\title{
SopB-Mediated Recruitment of SNX18 Facilitates Salmonella Typhimurium Internalization by the Host Cell
}

\author{
David Lieb/ ${ }^{1+}$, Xiaying $Q i^{1}$, Yang Zhe ${ }^{1}$, Timothy C. Barnett ${ }^{2,3}$ and Rohan D. Teasdale ${ }^{1,2,4 *}$ \\ ${ }^{1}$ Institute for Molecular Bioscience, The University of Queensland, Brisbane, QLD, Australia, ${ }^{2}$ Australian Infectious Diseases \\ Research Centre, The University of Queensland, Brisbane, QLD, Australia, ${ }^{3}$ School of Chemistry and Molecular Biosciences, \\ The University of Queensland, Brisbane, QLD, Australia, ${ }^{4}$ School of Biomedical Sciences, The University of Queensland, \\ Brisbane, QLD, Australia
}

OPEN ACCESS

Edited by:

Leigh A. Knodler,

Washington State University,

United States

Reviewed by:

Luís Jaime Mota,

Faculdade de Ciências e Tecnologia da Universidade Nova de Lisboa,

Portugal

Jost Enninga,

Pasteur Institute, France

*Correspondence: Rohan D. Teasdale r.teasdale@uq.edu.au

${ }^{\dagger}$ Present Address: David Liebl, Institute of Molecular and Cell Biology, Agency for Science, Technology and

Research, Proteos, Singapore

Received: 16 March 2017 Accepted: 31 May 2017

Published: 15 June 2017

Citation:

Liebl D, Qi X, Zhe Y, Barnett TC and Teasdale RD (2017) SopB-Mediated

Recruitment of SNX18 Facilitates Salmonella Typhimurium Internalization by the Host Cell.

Front. Cell. Infect. Microbiol. 7:257. doi: 10.3389/fcimb.2017.00257
To invade epithelial cells, Salmonella enterica serovar Typhimurium (S. Typhimurium) induces macropinocytosis through the action of virulence proteins delivered across the host cell membrane via a type III secretion system. We show that after docking at the plasma membrane $S$. Typhimurium triggers rapid recruitment of cytosolic SNX18, a SH3-PX-BAR domain sorting nexin protein, to the bacteria-induced membrane ruffles and to the nascent Salmonella-containing vacuole. SNX18 recruitment required the inositol-phosphatase activity of the Salmonella effector SopB and an intact phosphoinositide-binding site within the PX domain of SNX18, but occurred independently of Rho-GTPases Rac1 and Cdc42 activation. SNX18 promotes formation of the SCV from the plasma membrane by acting as a scaffold to recruit Dynamin-2 and N-WASP in a process dependent on the SH3 domain of SNX18. Quantification of bacteria uptake revealed that overexpression of SNX18 increased bacteria internalization, whereas a decrease was detected in cells overexpressing the phosphoinositide-binding mutant $\mathrm{R} 303 \mathrm{Q}$, the $\triangle \mathrm{SH} 3$ mutant, and in cells where endogenous levels of SNX18 were knocked-down. This study identifies SNX18 as a novel target of SopB and suggests a mechanism where $S$. Typhimurium engages host factors via local manipulation of phosphoinositide composition at the site of invasion to orchestrate the internalization process.

Keywords: Salmonella, macropinocytosis, sorting nexin, phosphoinositide, host-pathogen interaction

\section{INTRODUCTION}

To gain entry into non-phagocytic epithelial cells, various viral and bacterial human pathogens co-opt the endocytic machinery by modulation of phosphoinositide metabolism (PizarroCerda and Cossart, 2004) or actin cytoskeleton (Haglund and Welch, 2011). One of the most efficient routes for pathogens to enter the host cells is macropinocytosis, a distinct form of endocytosis characteristic for its non-selective and high turnover uptake of extracellular fluid into macropinosomes (Lim et al., 2008; Kerr et al., 2010).

Abbreviations: SNX18, Sorting nexin 18; S. Typhimurium, Salmonella enterica serovar Typhimurium; SL-mRFP, $S$. Typhimurium expressing mRFP; SCV, Salmonella-containing vacuole; Salmonella SPI1-T3SS, pathogenicity island 1-type three secretion system; PtdIns, Phosphatidylinositol; N-WASP, Neuronal Wiscott-Aldrich syndrome protein; MOI, Multiplicity of infection; PH domain, Pleckstrin homology domain. 
Salmonella are facultative intracellular Gram-negative bacteria, which infect and replicate within both epithelial cells and macrophages. To invade epithelial cells, Salmonella induces macropinocytosis at the site of entry (Francis et al., 1993) by translocating a set of effector proteins into the host cell cytoplasm via a type III secretion system (T3SS) encoded by Salmonella pathogenicity island 1 (SPI). Interactions between the translocated effector proteins and host cell targets result in orchestrated manipulation of phosphoinositide signaling, Rho-GTPase function and actin cytoskeleton remodeling that promotes internalization of the bacteria into a membranebound organelle, termed the Salmonella-containing vacuole (SCV) (Haraga et al., 2008). Although it has been reported that the biogenesis and release of the nascent SCV from the plasma membrane is mediated by the virulence protein SopB (Terebiznik et al., 2002), most likely in cooperation with SopD (Bakowski et al., 2007), the host-cell factors involved in this process remain largely unknown.

Salmonella enterica serovar Typhimurium (S. Typhimurium) has been used as a model organism to study the role of phosphoinositides in bacteria-mediated manipulation of the host endocytic trafficking pathways (Kerr et al., 2010). The phosphoinositides are key regulators of the actin cytoskeleton and membrane trafficking and are indispensable for macropinocytosis and maturation of the SCV (Kerr et al., 2012). Specific membrane bound phosphoinositides are essential for recruitment of sorting nexins, a family of Phox homology (PX) domain containing proteins that orchestrate membrane trafficking, cargo sorting, and endosomal recycling in coordination with other effectors including RabGTPases (Teasdale and Collins, 2012). Several sorting nexins (SNX), including SNX1 and SNX3, have been functionally linked to membrane tubulation, remodeling and maturation of the SCV during early stages of $S$. Typhimurium infection (Bujny et al., 2008; Braun et al., 2010) and our earlier studies also shown that SNX1 and SNX5 are essential for membrane recycling and macropinosome biogenesis in non-infected cells (Bryant et al., 2007; Lim et al., 2008). Our recent screen of SNX-PXBAR proteins involved in macropinocytosis identified another member of sorting nexin family, SNX18, which significantly increased the fluid phase uptake when overexpressed in epithelial cells (Wang et al., 2010).

The function of SNX18 is still not well-understood. Recent studies proposed that SNX18 might function as a paralog of SNX9 that is engaged in endosome formation and scission from the plasma membrane by coupling phosphoinositide recognition to actin assembly (Soulet et al., 2005; Pylypenko et al., 2007; Park et al., 2010). SNX18 and SNX9 are homologs with an identical SH3-PX-BAR domain modular structure and some conserved binding partners (Haberg et al., 2008; Park et al., 2010). However, variations between the SNX9 and SNX18 expression profile within different cell lines (Park et al., 2010), tissues (Haberg et al., 2008), or embryonic stages (Nakazawa et al., 2011), and differences in their affinity to particular membrane bound phosphoinositides (Haberg et al., 2008; Yarar et al., 2008) suggest that SNX18 and SNX9 may have evolved to perform different functions.
In this study, we investigate the function of SNX18 in Salmonella-driven exploitation of macropinocytosis during invasion of epithelial cells. We demonstrate that $S$. Typhimurium recruits SNX18, N-WASP and Dynamin-2 to the site of invasion through the phosphatidylinositol phosphatase activity of the T3SS effector SopB. We propose that modulation of phosphoinositides at the site of $S$. Typhimurium invasion recruits SNX18 as a scaffold to organize the molecular machinery required for formation and scission of the nascent SCV from the cell surface, which increases the efficiency of bacteria internalization.

\section{MATERIALS AND METHODS}

\section{Antibodies and Reagents}

The following primary antibodies were used: Rabbit polyclonal anti-SNX18 IgG (AbCam, ab99035); mouse monoclonal antimyc (Cell Signaling Technology, 2272); Rabbit polyclonal anti$\beta$-Tubulin (LI-COR Biosciences, 926-42211); mouse monoclonal anti- $\alpha$-tubulin Clone DM 1A (Sigma Aldrich, T9026), anti- $\alpha 1$ $\mathrm{Na}+/ \mathrm{K}+$ ATPase antibody (ab7671, Abcam), mouse monoclonal anti-LPS (Abcam, ab 8274); Mouse anti-Phospho-Tyrosine antibody, clone 4G10 (Millipore, 05-1050); mouse monoclonal anti-HA (Covance); Rabbit monoclonal anti-Akt (pan) C67E7 (Cell Signaling, 4691); and anti-phospho-Akt (Ser473) D9E (Cell Signaling, 4060). Goat anti-mouse coupled to Alexa Fluor 405, 546 or 647 (Invitrogen) were used as secondary antibodies for immunofluorescence. The IRDye800CW goat anti-mouse IgG; IRDye800CW donkey anti-rabbit IgG; IRDye680LT goat anti-rabbit IgG; and IRDye680LT donkey anti-mouse IgG were purchased from LI-COR Biosciences. Phalloidin-Alexa Fluor 635, fixable analog of lipophilic membrane stain FM 4-64FX and Wheat germ agglutinin (WGA) coupled to Alexa Fluor 647 were from Invitrogen (A34054, F34653, and W324666). Selective inhibitor of Rac1-GEF interaction NSC 23766 was purchased from Tocris Bioscience and the Akt1/2 kinase inhibitor from Sigma Aldrich.

\section{Plasmids and Constructs}

The Dynamin-2 encoding construct pCBDYN2-HA was obtained from Dr. S.L. Schmid (Scripps Research Institute, La Jolla, CA). The 2xFYVE-EGFP construct was generated as described by Pattni et al. (2001) and provided by F. Meunier (University of Queensland, Australia). The construct expressing EGFP-tagged $\mathrm{PH}$ domain of Akt was a gift from Prof. C. Mitchell (Monash University, Australia) and the PH domain of TAPP1 (aa 95400) for generation of EGFP-TAPP1-PH was a gift from Dr. S. Dowler (MRC, University of Dundee, Dundee, UK). The following expression vectors have been described previously: The pEGFP-C1-N-WASP (Lommel et al., 2001), the pcDNA3 expression vectors encoding constitutively active Rac1 or Cdc42 and dominant negative Rac1 or Cdc42 (Wang et al., 2005), the pEGFP-FERM (Marion et al., 2011), the pEGFP-SNX18 and pEGFP-SNX18: $\triangle$ SH3 (Wang et al., 2010), the Btk-PH-GFP (Varnai et al., 1999). The LifeAct-Ruby construct was a gift Roland Wedlich-Söldner, (Max Planck Institute of Biochemistry, Germany). 
The pEGFP-SNX18:R303Q was generated by site-directed mutagenesis from the full length pEGFP-SNX18. Cloning of Nterminal-myc-tagged $S$. Typhimurium effectors was performed by ligation-independent cloning (LIC) (Aslanidis and de Jong, 1990) into the plasmid pcDNA3.1-nMyc-LIC, constructed as follows: A DNA cassette encoding a Myc tag, LIC sequences, chloramphenicol acetyl transferase (cat), and $c c d B$ was constructed by PCR using primers N-Myc-catccdB-NheI$S$ and catccdB-ApaI-A and Reading Frame Cassette A template DNA from the Gateway Vector Conversion System (Life Technologies); The resulting PCR product was digested with NheI-ApaI and ligated into NheI-ApaI-digested pcDNA3.1(+). The resulting plasmid, pcDNA3.1-nMyc-LIC, was maintained in E. coli $c c d B$ Survival ${ }^{\mathrm{TM}} 2 \mathrm{~T}^{\mathrm{R}}$ cells (Life Technologies). For LIC reactions, pcDNA3.1-nMyc-LIC was digested with EcoRV and treated with T4 DNA polymerase in the presence of dCTP to generate linearized vector with single-stranded DNA overhangs. The genes encoding individual $S$. Typhimurium effectors were amplified by PCR using primers which incorporated 5' and 3' LIC sequences and S. Typhimurium SL1344 template DNA. Each PCR product was treated with T4 DNA polymerase in the presence of dGTP to generate single-stranded overhangs. The polymerase-treated vector and effector DNA fragments were combined on ice and transformed into chemically-competent $E$. coli $\mathrm{DH} 5 \alpha$. Vectors encoding Myc-tagged phosphatase inactive SopB mutants SopB:C460S, R466A, and K528A were constructed by PCR amplification using pcDNA3.1 (+) vector encoding Myc-tagged SopB (wild type) as a template. All mutants were constructed using the QuikChange XL-site directed mutagenesis kit (Stratagene) according to manufacturer's instructions, and sequences were confirmed by direct DNA sequencing at AGRF (Australian Genome Research Facility). All primers used in this study are listed in Table $\mathbf{1 .}$

For complementation of SopB in $S$. Typhimurium $\triangle s o p B$ mutant bacteria, the coding sequence of the wild type $\operatorname{sop} B$ and that of the C460S mutant of $s o p B$ were amplified by PCR using pcDNA3.1 (+) vector encoding Myc-tagged SopB (wild type) or Myc-tagged C460S mutant of SopB as templates. Corresponding primers used for the PCR are listed in Table 1. The PCR products were digested with EcoRI and XhoI and subcloned into pWSK29 vector (GenBank: AF016889.1).

\section{Cell Culture, Transfections, and Generation of SNX18 Knockdown}

Human epithelial HEK293 cells (CRL-1573) and mouse macrophages RAW264.7 (TIB-71) were grown in complete DMEM medium (Life technologies) supplemented with $10 \%(\mathrm{v} / \mathrm{v})$ FCS. Cells were transfected using Lipofectamine 2000

TABLE 1 | Primers used in this study.

\begin{tabular}{|c|c|}
\hline Primer & Sequence $^{*}$ \\
\hline N-Myc-catccdB-Nhel fwd & $\begin{array}{l}\text { 5'-GCATGCTAGCCACCATGGAGCAGAAGCTGATAAGTGAGGAGGATATCAGGCA } \\
\text { CCCCAGGCTITACACTITATGCTTC-3' }\end{array}$ \\
\hline catccdB-Apal rev & 5'-GCATGGGCCCGACATGAAGGTTAGGGATATCGACCTGCAGACTGGCTGTGTATA AG-3' \\
\hline sipA fwd & 5'-TGATAAGTGAGGAGGATCTGGTTACAAGTGTAAGGACTCAGC-3' \\
\hline sipA rev & 5’-ATGATGGTTAGGGATCTTAACGCTGCATGTGCAAGCCATC-3' \\
\hline sipC fwd & 5’-TGATAAGTGAGGAGGATCTGTTAATTAGTAATGTGGGAATAAATC-3' \\
\hline sipC rev & 5’-ATGATGGTTAGGGATCTTAAGCGCGAATATTGCCTGCGAT-3' \\
\hline sopB fwd & 5'-TGATAAGTGAGGAGGATCTGCAAATACAGAGCTTCTATCACTC-3' \\
\hline sopB rev & 5’-ATGATGGTTAGGGATCTCAAGATGTGATTAATGAAGAAATGCCTITACTG-3' \\
\hline sopD fwd & 5’-TGATAAGTGAGGAGGATCTGCCAGTCACTITAAGCTTCGGTAATCATC-3' \\
\hline sopD rev & 5’-ATGATGGTTAGGGATCTTATGTCAGTAATATATTACGACTGCACCCATC-3' \\
\hline sopE2 fwd & 5'-TGATAAGTGAGGAGGATCTGACTAACATAACACTATCCACCCAGCAC-3' \\
\hline sopE2 rev & 5'-ATGATGGTTAGGGATCTCAGGAGGCATTCTGAAGATACTTATTC-3' \\
\hline sptP fwd & 5'-TGATAAGTGAGGAGGATCTGCTAAAGTATGAGGAGAGAAAATTGAATAATTAA ACGTTG-3' \\
\hline sptP rev & 5'-ATGATGGTTAGGGATCTCAGCTTGCCGTCGTCATAAG-3' \\
\hline SopB:C460S fwd & 5'-GGTACCCGCCTGGAATAGTAAAAGCGGCAAAG-3' \\
\hline SopB:C460S rev & 5'-CTITGCCGCTITACTATTCCAGGCGGGTACC-3' \\
\hline SopB:R466A fwd & 5’-CCTGGAATTGTAAAAGCGGCAAAGATGCTACAGGGATGATGG-3' \\
\hline SopB:R466A rev & 5'-CCATCATCCCTGTAGCATCTITGCCGCTITACAATTCCAGG-3' \\
\hline SopB:K528A fwd & 5'-GGGCGGGAAACAAAGTAATGGCAAATITATCGCCAGAGGTGC-3' \\
\hline SopB:K528A rev & 5'-GCACCTCTGGCGATAAATTTGCCATTACTTTGTITCCCGCCC-3' \\
\hline SNX18:R303Q fwd & 5'-CCAGGTGCCCGTGCACAGGCAATATAAGCACTTCGATTGG-3' \\
\hline SNX18:R303Q rev & 5'-CCAATCGAAGTGCTTATATTGCCTGTGCACGGGCACCTGGG-3' \\
\hline sopB/sopB:C460S fwd & 5'-GCATGAATTCTATTCAGGAATATTAAAAACGCTATGCAAATACAGAGCTTCTATCACTC-3' \\
\hline sopB/sopB:C460S rev & 5'-GCATCTCGAGTACCTCAAGACTCAAGATGTGATTAATGAAGAAATGCCTIITACTG-3' \\
\hline
\end{tabular}


(Invitrogen). For stable expression, transfected cells were selected with $400 \mu \mathrm{g} / \mathrm{ml}$ Geneticin (G418), and cell lines were generated by limit dilution. To generate the shRNA-mediated knockdown of SNX18, the pGIPZ-shRNAmir clones (V2LHS_184681, V2LHS_37858, V2LMM_58706) complementary to human SNX18 were obtained from Thermo Scientific. HEK293 cells were transfected with pGIPZ constructs using Lipofectamine 2000 (Invitrogen) and non-silencing shRNA was transfected as a control. Cells were split $24 \mathrm{~h}$ post transfection and selected in $1 \mu \mathrm{g} / \mathrm{ml}$ puromycin for 3 or more days before SNX18 protein levels were tested by western blot. Cells were then transfected as above, selected with $1 \mu \mathrm{g} / \mathrm{mL}$ puromycin for 7 days to generate stable cell lines. Cells stably expressing non-silencing shRNA were used as a control knockdown.

\section{Bacteria Strains and Infections}

Wild type S. Typhimurium strain SL1344 expressing pBRmRFP.1 (Birmingham et al., 2006) or pFPV25.1-GFP (Knodler et al., 2005) were used in this study. The isogenic $\Delta$ sopB mutant has been described earlier (Steele-Mortimer et al., 2000) and provided by Dr. N. Brown (Department of Microbiology and Immunology; University of Melbourne; Australia). The $S$. Typhimurium SL1344 mutants $\triangle i n v A$ (SPI1-T3SS deficient) and $\triangle s s a R$ (SPI2-T3SS deficient) were provided by Prof. R. Strugnell (University of Melbourne, Australia) (Kupz et al., 2012). Where non-fluorescent bacteria were utilized, the mouse monoclonal anti-LPS antibody (Abcam) was used for immunofluorescent detection. To prepare invasive (SPI1-T3SS activated) bacteria, the overnight culture was subcultured 1:60 in LB medium and grown for another $4 \mathrm{~h}$ to reach late log phase. Bacteria were washed three-times in Hanks buffered salt solution (HBSS) and diluted in serum-free DMEM medium (for immunofluorescence) or in $\mathrm{CO}_{2}$-independent imaging medium (Invitrogen) for live imaging. For complementation of SopB in $S$. Typhimurium $\triangle \operatorname{sop} B$ mutant bacteria, the sequence verified plasmids were transformed into electrocompetent $S$. Typhimurium $\Delta s o p B$ mutant bacteria by electroporation using Bio Rad Gene Pulser II Electroporation System and positive clones of complemented $S$. Typhimurium were selected using kanamycin.

\section{Electron Microscopy}

The HEK293 cells expressing EGFP-SNX18 were infected (or mock infected) with $S$. Typhimurim (SL1344) for $10 \mathrm{~min}$, fixed with $4 \%(\mathrm{w} / \mathrm{v})$ formaldehyde, and $0.1 \%$ glutaraldehyde $(\mathrm{w} / \mathrm{v})$ in $0.1 \mathrm{M}$ phosphate buffer and embedded in $10 \%(\mathrm{w} / \mathrm{v})$ gelatin (Sigma). Solidified gelatin blocks were infiltrated with $2.3 \mathrm{M}$ sucrose, mounted on aluminum pins and frozen in liquid nitrogen. Ultrathin sections were cut on Leica EM UC6 cryotome, collected on a drop of sucrose-methylcellulose and transferred on carbon-coated CuPd EM grids with formvar film. EGFPSNX18 was detected on sections by rabbit anti-GFP antibody (Molecular Probes) followed by protein A coupled with 10 $\mathrm{nm}$ gold (CMC Utrecht). Grids were contrasted, embedded in mixture of uranyl acetate-methylcellulose and analyzed with JEOL 1011 electron microscope operating at $80 \mathrm{kV}$. Micrographs were acquired by microscope-mounted CCD camera and ImageJ software (version 1.46a) was used for contrast adjustment and cropping of the final images. Manual quantification of gold marker localization was performed on micrographs by scoring each gold particle in a set of images for localization in plasma membrane or in cytosol.

\section{Subcellular Fractionation and Western Blotting}

HEK293 cells expressing EGFP-tagged SNX18 were serum starved overnight before bacterial infection at MOI $=100$ or mock infection. At 10 min post infection, cells were washed twice with ice-cold PBS and harvested for subcellular fractionation using a modification of the protocol described by Piper et al. (1991). Briefly, cells were resuspended in HES buffer $(20 \mathrm{mM}$ HEPES, pH 7.4, $0.25 \mathrm{mM}$ sucrose, $1 \mathrm{mM}$ EDTA, $10 \mathrm{mM}$ sodium pyrophosphate, $30 \mathrm{mM}$ sodium vanadate, $0.5 \mathrm{mM}$ AEBSF and protease inhibitor cocktail) and subjected to homogenization by using $22 \mathrm{G} \times 3 / 4$ needle. Resulting suspensions were centrifuged at $17,200 \mathrm{~g}$ for $20 \mathrm{~min}$ to generate crude plasma membrane (PM) fraction and a fraction containing cytosol and microsomes. The cytosol fraction was then purified by microsome sedimentation at $180,000 \mathrm{~g}$ for $60 \mathrm{~min}$. Crude PM fractions were washed once and resuspended in HES buffer, and then layered onto $1.12 \mathrm{M}$ sucrose cushion, containing $20 \mathrm{mM}$ HEPES, pH 7.4, $1.12 \mathrm{M}$ sucrose and $1 \mathrm{mM}$ EDTA, followed by centrifugation at 100,000 g for $1 \mathrm{~h}$ to yield a white interface, which contains the purified $\mathrm{PM}$ fraction. The white interface was mixed with HES buffer, and centrifuged at 125,000 $\mathrm{g}$ for $30 \mathrm{~min}$ to pellet the purified PM fraction. For protein electrophoresis, $30 \mu \mathrm{g}$ of total protein of whole cell lysate or cytosol fractions or $15 \mu \mathrm{g}$ of total protein of the plasma membrane fractions were loaded on to $8 \%(\mathrm{w} / \mathrm{v})$ SDSPAGE gel and transferred onto a PVDF membrane (ImmobilonFL, Millipore) according to manufacturer's instructions. Western immunoblots were performed with anti-SNX18, anti- $\alpha$-Tubulin and anti- $\mathrm{Na}+/ \mathrm{K}+$ ATPase primary antibodies in Odyssey blocker, followed by IRdye800 anti-rabbit secondary antibody, and membranes were scanned using Odyssey Infrared Imaging System (LI-COR Biosciences) according to manufacturer's instructions. The integrated intensity of each band of interest was measured by Odyssey software.

\section{AKT Inhibitor Treatment and S. Typhimurium Infection}

Overnight serum-starved HEK293 cells were pre-treated with $10 \mu \mathrm{M}$ AKTil/2 (Sigma-Aldrich) for $30 \mathrm{~min}$ before infection with $S$. Typhimurium (wild type or $\triangle s o p B$ ). At 10 min post infection, cells were washed with ice-cold PBS and harvested in lysis buffer (50 mM HEPES (pH 7.4), 1\% (v/v) Triton $\mathrm{x}-100,150 \mathrm{mM}$ sodium chloride, $1 \mathrm{mM}$ EDTA, $10 \mathrm{mM}$ sodium pyrophosphate, $30 \mathrm{mM}$ sodium vanadate, $0.5 \mathrm{mM}$ AEBSF and protease inhibitor cocktails). Cell lysates were cleared by centrifugation at $16,000 \mathrm{~g}$ for $10 \mathrm{~min}$ and protein concentrations were quantified by BCA protein assay. Equal amounts of cell lysates were subjected to SDS-PAGE and immunoblotting, staining with anti-phospho $\mathrm{Ser}^{473}$ Akt, anti-Akt, or anti-Tubulin antibodies, followed by IRdye 800 anti-rabbit or IRdye680 anti-mouse secondary 
antibodies. Fluorescence intensities were detected using the LICOR Biosciences Odyssey Infrared Imaging system.

\section{Immunofluorescence}

For immunofluorescence, cells were grown on poly-L-lysine (PLL)-coated glass coverslips, fixed with $4 \%(\mathrm{w} / \mathrm{v})$ formaldehyde in $250 \mathrm{mM}$ HEPES and permeabilized with Triton X-100 $(0.25 \%$ $(\mathrm{v} / \mathrm{v})$ in PBS) before incubation with antibodies. Where nonfluorescent bacteria were used, cell permeabilization was required prior to bacteria immunodetection with anti-LPS antibody. For detection of extracellular and/or incompletely internalized mRFP-expressing bacteria, cells were incubated with the antiLPS antibody prior to cell permeabilization. Cells on coverslips were incubated with primary antibodies in $0.25 \%(\mathrm{w} / \mathrm{v})$ BSA in PBS for $1 \mathrm{~h}$, followed by wash in PBS and $1 \mathrm{~h}$ incubation with secondary antibodies with or without DAPI and fluorescent Phalloidin (all in $0.25 \%$ (w/v) BSA in PBS). After washing in PBS, coverslips were rinsed in water and mounted on glass slides using Dako Fluorescent Mounting Medium. Fluorescent images were acquired using confocal microscope Zeiss LSM 710 with PlanApochromatic 63x/1.4 Oil DIC objective, operated by ZEN2000 acquisition software, or using fluorescence microscope Olympus DP-71 with Plan-Apochromatic 10x/0.40 and 20x/0.75 objective, equipped with $12 \mathrm{Mp}$ Color Camera and images acquired using DP Capture and DP Manager software.

\section{Live Cell Imaging}

Cells were grown on glass bottom chamber slides (Lab-Tek, Thermo Scientific), and the culture medium was replaced with $\mathrm{CO}_{2}$-independent medium (Invitrogen) prior to imaging. To examine the interaction between bacteria and the host cells from the onset of infection, invasive (SPI1-T3SS-induced) bacteria were pre-diluted in $\mathrm{CO}_{2}$-independent medium and added directly to the chamber slide "on stage" during timelapse imaging. The confocal z-stacks or time-lapse of single confocal sections were acquired typically during the first $10 \mathrm{~min}$ of infection at a frequency of $6 \mathrm{~s}$ between frames and at the maximal resolution of $70 \mathrm{~nm}$ of pixel size. A Zeiss LSM 710 FCS inverted confocal microscope operated by ZEN2009 acquisition software and equipped with a temperature controlled incubation chamber and Plan-Apochromat 63x/1.4 Oil DIC objective was used for imaging.

\section{Image Processing and Image Analysis}

ImageJ software (version 1.46a) was used for fluorescent channels splitting or merging, image area cropping, orthogonal or maximum z-stack projections, time stamper insertions, montage of selected frames, RGB line profiling, and brightness and contrast adjustment of raw acquisition images where required. To analyze the kinetics of protein recruitment or depletion, the increase or decrease of EGFP and mCherry fluorescence intensity was assessed by stack-measurement of integrated fluorescence density of selected ROI (all identical size) after the RGB image was split in separate eight-bit fluorescent channels. Absolute values were normalized to frame 1 for both channels to demonstrate the fold of fluorescence increase/decrease over time of the sequence. Corresponding ROIs in non-infected cell (on the same movie) were selected as a control for fluorescence fluctuations and bleaching. The co-localization between SNX18 and phosphoinositide binding probes has been analyzed by co-localization highlighter plugin in (ImageJ) and the total co-localization area in each time point of the movie was quantified by particle analysis. Co-localization between SNX18 and Dynamin-2 or N-WASP within bacteria-containing membrane ruffles was quantified by co-localization threshold plugin in ImageJ. From 10 to 20 cells or regions of interest (ROI) per each sample were analyzed. To demonstrate an increase or decrease of co-localization relative to controls, statistics were calculated from values that represent the percentage of voxels which have both channel 1 and channel 2 intensities above threshold, expressed as a percentage of the total number of pixels in the image.

\section{Image-Based Quantification of Macropinocytosis and Bacterial Uptake}

The rate of macropinocytosis was assessed by fluorescentdextran uptake assay. Briefly, cells were grown on PLL-coated coverslips and starved $\mathrm{O} / \mathrm{N}$ in FCS-free DMEM medium before adding $100 \mathrm{ug} / \mathrm{ml}$ of anionic, fixable Dextran (MW 10000) conjugated to Alexa Fluor 647 with $10 \mathrm{ng} / \mathrm{ml}$ of EGF. After $10 \mathrm{~min}$ of dextran uptake, cells were fixed with $4 \%(\mathrm{w} / \mathrm{v})$ formaldehyde in $250 \mathrm{mM}$ HEPES and mounted on coverslips for imaging using fluorescent mounting medium (Dako). For the bacterial uptake assay, stable SNX18 knockdown cells (RFPpositive) and SNX18 overexpressing cells (EGFP-positive) were grown as above and infected with EGFP or RFP-expressing $S$. Typhimurium respectively, at an $\mathrm{MOI}=10$. At $10 \mathrm{~min}$ post infection, cells were washed, fixed and mounted for imaging as described above. Macropinosomes and intracellular bacteria were quantified using ImageJ software for image analysis as described previously (Wang et al., 2010). Briefly, RGB images were split to eight-bit channels (EGFP and RFP), subjected to segmentation by thresholding, cell mask subtraction by image calculator (to exclude extracellular dextran aggregates or noninternalized bacteria) and particle analysis function was then used to measure counts, integrated density and area of spots. Original images were acquired under the same conditions for all samples, batch processing was performed using Image J Macro and about $250 \sim 300$ cells per sample were quantified for statistical evaluation.

\section{RESULTS}

\section{SNX18 Is Recruited to the Plasma} Membrane during S. Typhimurium Invasion We reported recently (Wang et al., 2010) that SNX18 was the most potent of the three SH3-PX-BAR sorting nexins in increasing the EGF-stimulated macropinocytosis when transiently overexpressed in epithelial cells. Because macropinocytosis is targeted by $S$. Typhimurium, we first aimed to determine the role of SNX18 in S. Typhimurium invasion of epithelial cells by assessing the rate of macropinocytosis and efficiency of bacterial internalization in human embryonic kidney 

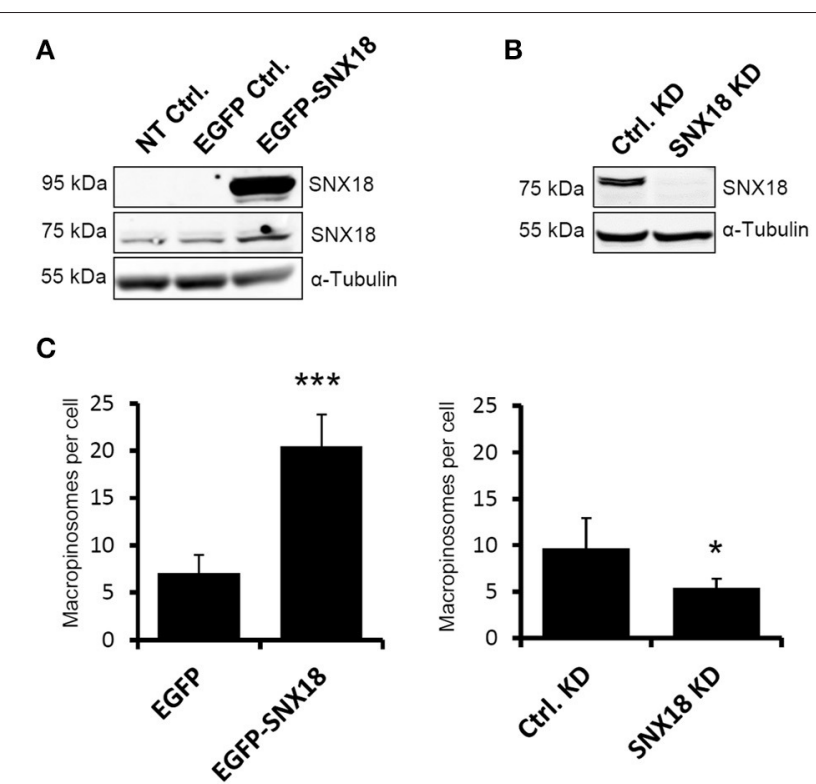

D
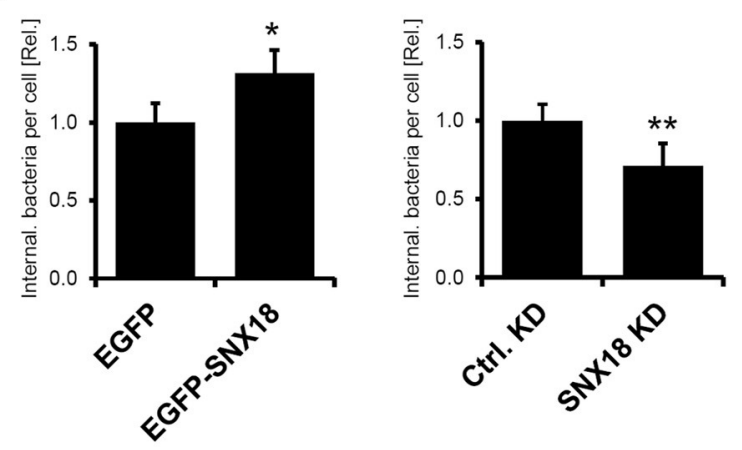

FIGURE 1 | SNX18 functions in macropinocytosis and S. Typhimurium internalization. (A) Protein levels of endogenous SNX18 $(75 \mathrm{kDa})$ and EGFP-SNX18 (95 kDa) in non-transfected HEK293 cells and in cells overexpressing EGFP or EGFP-SNX18 were detected by western blot using anti-SNX18 antibody. (B) Protein levels of endogenous SNX18 in cells 6 days post induction of shRNA-mediated knockdown and in control (non-induced) cells. $\alpha$-Tubulin was used as a loading control. (C) The rate of

macropinocytosis in cells overexpressing SNX18 (left) and in SNX18 knockdown cells (right) assessed by fluorescent dextran uptake assay in EGF-treated HEK293 cells. Numbers of dextran-positive macropinosomes per cell were counted by image analysis. The average values in control cells were normalized to one to demonstrate the fold change in EGFP-SNX18 expressing cells and SNX18 knockdown cells. Between 250 and 300 cells per sample were analyzed. Bars indicate the mean + standard deviation between three experiments. ${ }^{* * *} p<0.001,{ }^{*} p<0.05, t$-test. (D) Efficiency of bacteria internalization in cells overexpressing SNX18 (left) and in SNX18 knockdown cells (right). The amounts of intracellular bacteria per cell were quantified at 10 min post infection using image analysis with exclusion of extracellular bacteria (labeled by anti-LPS antibody). The average values in control cells were normalized to one to demonstrate the fold change in EGFP-SNX18 expressing cells and SNX18 knockdown cells. Bars indicate the mean + standard deviation between three experiments, ${ }^{*} p<0.05,{ }^{* *} p<0.01, t$-test.

epithelial cells (HEK293) overexpressing EGFP-SNX18 and in HEK293 cells where endogenous levels of SNX18 were reduced by inducible shRNA-mediated knockdown. In EGFP-SNX18 transfected cells the SNX18 expression levels (Figure 1A) were $\sim 20$-fold higher than endogenous levels (data not shown), while the endogenous levels of SNX18 in the stable SNX18 shRNA cell line were below detection 6 days post induction of shRNA expression (Figure 1B).

To analyze the effect of SNX18 expression levels on macropinocytosis, we measured the rate of macropinocytosis in each cell line by dextran uptake assay using fluorescently labeled dextran. Quantification of dextran-positive macropinosomes (integrated fluorescent density) in EGF-treated cells after $10 \mathrm{~min}$ of dextran uptake revealed 3-fold increase in cells expressing EGFP-SNX18 in comparison to controls, and a 2-fold decrease in SNX18 knockdown cells relative to control knockdown (Figure 1C).

Next we investigated whether overexpression or knockdown of SNX18 affects internalization of S. Typhimurium. Cells were infected with mRFP-expressing $S$. Typhimurium (SLmRFP) and the amount of fully internalized bacteria was determined at 10 min post infection by quantitative fluorescence microscopy. Extracellular bacteria were differentiated from internalized bacteria by immunostaining of non-permeabilized cells with anti-LPS antibody. The amount of internalized bacteria was $32 \pm 6 \%$ higher in EGFP-SNX18 expressing cells when compared to EGFP-expressing control cells, while knockdown of SNX18 decreased the amount of intracellular bacteria by $29 \pm 9 \%$ relative to controls (Figure 1D). Thus, although depletion of SNX18 did not completely abolish macropinocytosis or bacteria internalization, modulation of SNX18 levels resulted in significant impact on both macropinocytosis and $S$. Typhimurium internalization. In order to minimize the influence of passive uptake of bacteria from the media, we analyzed short-lasting but synchronized (simultaneous) invasion at relatively high MOI, rather than prolonged invasion at low MOI, that would imply consecutive (and potentially passive) invasion events. For this we reduced bacteria adhesion/invasion time to $10 \mathrm{~min}$ only and optimized the MOI so that at end point of our invasion assay each cell internalized on average 1-3 bacteria $(\mathrm{MOI}=10)$ for quantification assay.

We next examined the subcellular localization and kinetics of EGFP-SNX18 in live HEK293 epithelial cells and RAW264.7 macrophages. Both of these cell types were selected as they undergo constitutive macropinocytosis with the majority of cells having numerous filopodia and also peripheral membrane regions exhibiting "ruffling." This enables monitoring the capacity of $S$. Typhimurium to elevate the local recruitment of proteins like SNX18, within the modified plasma membrane regions. During transient expression in non-infected cells, EGFP-SNX18 localization varied with protein expression levels, where high levels led to formation of elongated, thickened and often branched membrane tubules described previously in cells transfected with myc-tagged PX-BAR construct of SNX18 (Haberg et al., 2008). Therefore, for further analysis we isolated cells expressing moderate levels of exogenous SNX18, where EGFP-SNX18 localized to the cytosol but a fraction was readily associated with endosomes and with short, endosome-derived tubules usually concentrated at the base of the cell where EGFP-SNX18 co-localized with fluorescent lipophilic vital stain FM4-64 (Figure S1). Upon infection with S. Typhimurium, 
we observed a dramatic relocation of EGFP-SNX18 from the cytosol to the plasma membrane within 10 min post infection. A similar extent of SNX18 relocation was detected in RAW264.7 macrophages (Figure 2A) and in other human epithelial lines, including HeLa, MCF-7, and A431 (not shown), suggesting that Salmonella-mediated engagement of SNX18 is utilized in multiple cell types including those that do not undergo constitutive macropinocytosis like HeLa cells.

To investigate the dynamics of SNX18 recruitment to the plasma membrane during $S$. Typhimurium internalization, we performed live imaging of EGFP-SNX18-expressing HEK293 cells within the first $10 \mathrm{~min}$ of infection. Following docking of bacteria to the plasma membrane at the site of invasion, we observed a rapid burst of SNX18 recruitment within membrane ruffles when compared with non-infected cells, where a transient increase of SNX18 was detected only on the nascent macropinosomes. (Figure 2B and Movie 1). To examine the kinetics of SNX18 recruitment during early stages of infection, we next measured the amount of EGFP-SNX18 at the plasma membrane and cytosol at various time points post infection by quantitating the fluorescence density profiles of selected regions of interest. As shown in Figure 2B, the amount of EGFP-SNX18 at the plasma membrane rapidly increased 1.7fold within the first minute of infection while the concentration (fluorescence density) of the cytosolic pool of SNX18 within the same cell decreased. In contrast, insignificant changes in fluorescent density of EGFP-SNX18 in both, plasma membrane and cytosol were observed in non-infected cells.

To further examine the bacteria-induced recruitment of SNX18 to the plasma membrane at site of invasion, we infected (or mock-infected) the EGFP-SNX18 expressing cells and analyzed localization of this construct by electron microscopy using immunogold labeling on cryosections. In mock-infected cells, the gold-coupled marker of EGFP-SNX18 localized predominantly to the cytosol with only minor fraction $(24 \pm$ $4.5 \%)$ found in plasma membrane, while in infected cells, the majority $(58 \pm 5.5 \%)$ of the marker associated with the plasma membrane of bacteria-containing membrane ruffles and with the SCV during its closure at the cell periphery. In contrast, fully internalized SCV were devoid of SNX18 (not shown), suggesting that association of SNX18 with the SCV is only transient and occurs during the very early stage of the SCV formation (Figure 2C).

To confirm that also endogenous SNX18 is recruited to the plasma membrane the levels of cytosolic and membrane-bound SNX18 during bacteria internalization was then examined by cell fractionation and immunoblotting with anti-SNX18 antibodies. In agreement with the above results, the protein levels of membrane-bound endogenous SNX18 increased within the first 10 min of infection (Figure 2D) indicating that the presence of the EGFP-tag on SNX18 is not responsible for the induced recruitment to membranes observed during $S$. Typhimurium invasion in live cells.

We thus identified SNX18 within two fractions: a mobile, cytosolic fraction, and a membrane-bound fraction. Furthermore, we show that this ratio rapidly changes when SNX18 is recruited to the site of bacteria internalization. The kinetics of bacteria-induced SNX18 recruitment and the details of SNX18 localization within bacteria-containing membrane ruffles suggested that SNX18 is likely involved in the actin-driven remodeling of the plasma membrane which promotes formation of the nascent SCV.

\section{SNX18 Functions as a Scaffold for Molecular Machinery Driving Formation and Scission of the Nascent SCV}

The dynamics of SNX18 recruitment to the site of bacteria entry was next determined by live imaging at a higher spatiotemporal resolution. We observed a rapid increase in SNX18 at the site of bacterial docking, followed by recruitment of SNX18 to the edge of extending membrane ruffles that folded back to enclose bacteria, and continuous accumulation of SNX18 was then detected in the membrane of the nascent SCV during its formation and scission from the plasma membrane. The local increase in EGFP-SNX18 fluorescence density was detected within 10-20 s following bacterial contact with the cell surface and the process of internalization and SCV scission was completed within $1 \mathrm{~min}$. Subsequently, SNX18 rapidly depleted from the fully internalized SCV (Figure 3A and Movie 2).

Next we addressed the possibility that SNX18 functions in actin-driven membrane reorganization leading to bacterial confinement within closing membrane ruffles and scission of the nascent SCV from the plasma membrane. To visualize actin dynamics during bacteria internalization, the EGFP-SNX18 expressing cells were transfected with LifeAct-Ruby, a fluorescent 17-amino acid peptide which detects filamentous actin (F-actin) structures in live cells (Riedl et al., 2008), and infected with non-fluorescent $S$. Typhimurium in the presence of fluorescently coupled wheat germ agglutinin, which selectively binds to plasma membrane. Live imaging of these cells revealed that upon contact of bacteria with the cell surface, the burst of SNX18 correlates with a burst of actin polymerization during closure of the membrane ruffle around the nascent SCV and during SCV scission from the plasma membrane (within the first min of bacteria internalization). Once the SCV was fully internalized, SNX18 rapidly dissociated from the SCV followed by depolymerization of the F-actin coat (Figure 3B).

Recently it has been reported that SNX18 directly interacts with proteins required for endosome scission, Dynamin-2 and N-WASP (Park et al., 2010). Importantly, both of these proteins are also involved in the internalization of $S$. Typhimurium (Unsworth et al., 2004; Veiga et al., 2007). To determine whether $S$. Typhimurium can recruit Dynamin-2 and N-WASP to the site of invasion via SNX18, we analyzed colocalization of SNX18 with Dynamin-2 and N-WASP in cells during bacteria internalization. Substantial co-localization of SNX18 with both Dynamin-2 and N-WASP was found within bacteria-containing membrane ruffles and on the nascent SCV (Figures 4A,B). However, a significantly lower degree of co-localization was found in cells that co-expressed Dynamin-2 or N-WASP with SNX18 mutant lacking the N-terminal SH3 domain (SNX18: $\triangle \mathrm{SH} 3$ ). Although the SNX18: $\triangle \mathrm{SH} 3$ was recruited into Salmonellainduced membrane ruffles within $10 \mathrm{~min}$ of infection to a 
A
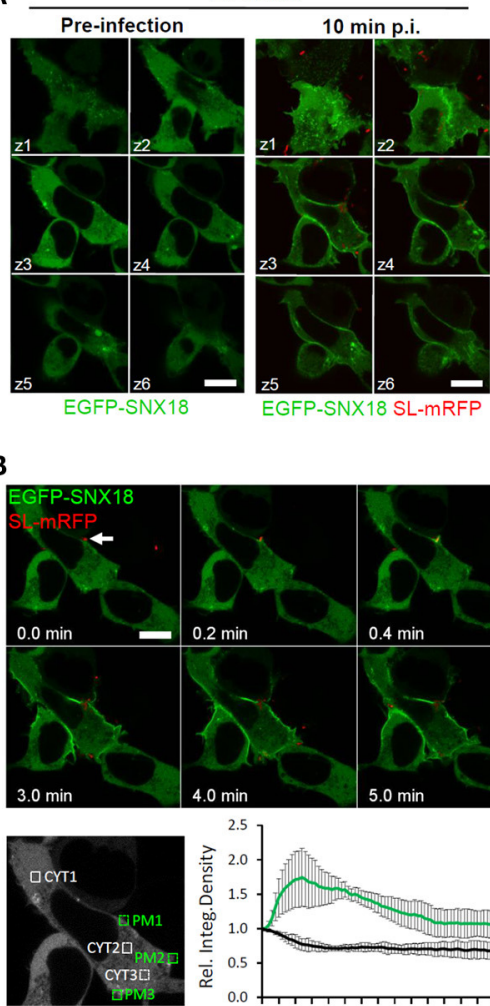

Infected (SL+)
EGFP-SNX18 SL-mRFP

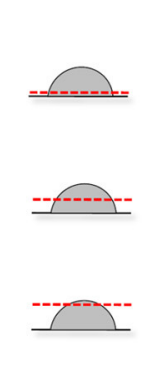

RAW264.7

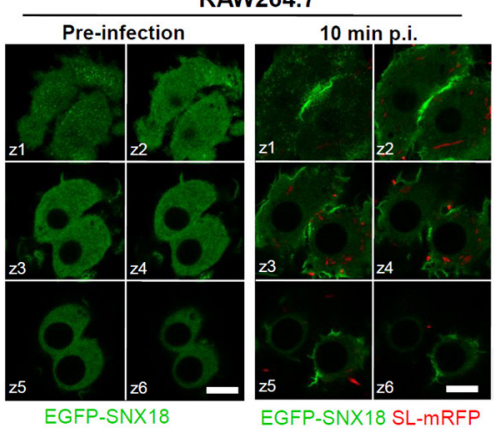

0-10 min p. i. (time-lapse)

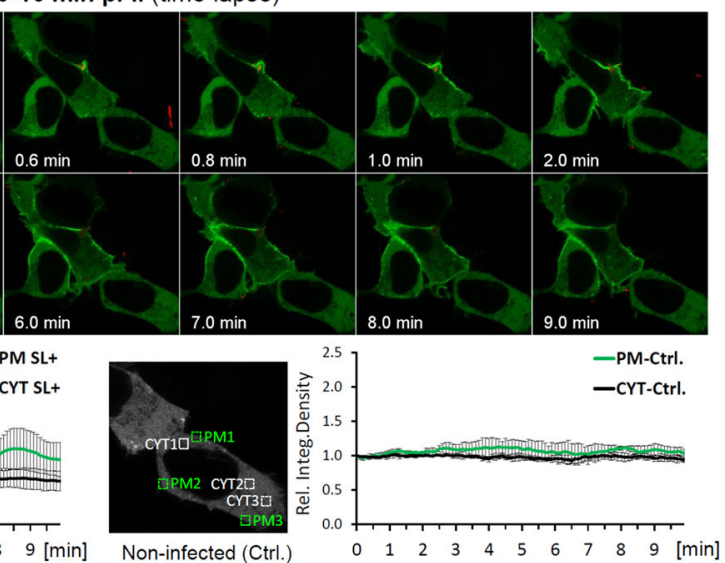

C
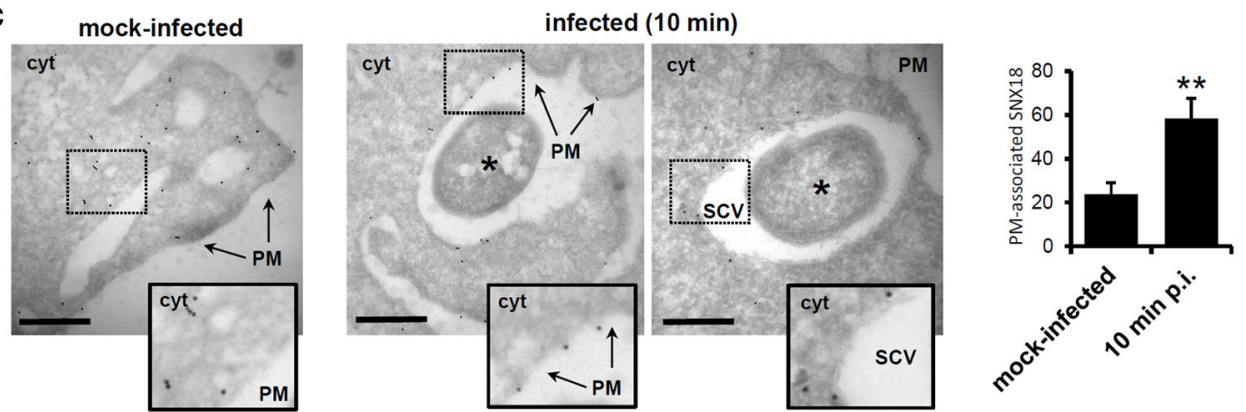

D

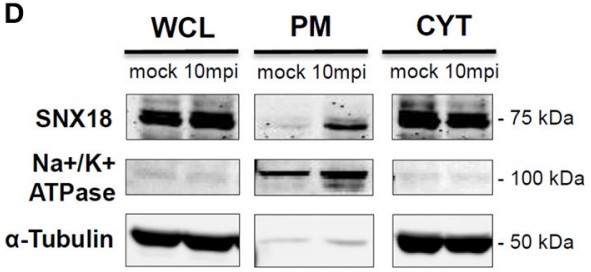

FIGURE 2 | Cytosolic SNX18 is recruited to the site of bacterial invasion at the plasma membrane. (A) Within 10 min of infection, the cytosolic fraction of EGFP-SNX18 is recruited to the plasma membrane in both, epithelial cells (HEK293) and macrophages (RAW264.7). Shown is a montage of confocal z-stack sections through live cells expressing EGFP-SNX18 pre-infection and 10 min post infection with SL-mRFP. Bars $=10 \mu \mathrm{m}$. (B) Kinetic measurements of SNX18 relocation to the plasma membrane. Upper panel: Series of confocal images from a time-lapse acquisition of HEK293 cells expressing EGFP-SNX18 during 10 min of infection with SL-mRFP (initial docking site of bacteria indicated by an arrow); Lower panel: Quantification of fluorescence (relative integrated density) within selected ROI at plasma membrane and in cytosol of infected (left) and adjacent non-infected control cell (right). Absolute values of integrated density at $\mathrm{T}=0 \mathrm{~min}$ post infection were normalized to 1 to demonstrate fold-increase/decrease in following time points. (C) Electron micrographs of frozen-hydrated sections through HEK293 cells with stable expression of EGFP-SNX18 fixed 10 min post infection (or mock-infection). The EGFP-SNX18 was detected by anti-GFP antibody followed by 10 nm Protein 
FIGURE 2 | Continued

A-Gold. The marker of SNX18 localizes primarily to the cytosol (cyt) in mock infected cells, while upon infection the SNX18 associates mainly with the plasma membrane (PM) and the membrane of the nascent SCV. Bacteria indicated by asterisk. Bar $=1 \mu \mathrm{m}$. Quantification of gold markers associated with plasma membrane in mock-infected cells and cells 10 min p.i. as a percentage of total gold markers per each image analyzed. $N=10, p=0.002$ ( $t$-test). (D) Endogenous SNX18 is also recruited to the plasma membrane during bacteria internalization: Whole cell lysate (WCL), plasma membrane (PM), and cytosolic (CYT) fractions from cells harvested at 10 min post infection (10 mpi) or mock-treatment (as a control). Endogenous SNX18 was detected on Western blot by anti-SNX18 antibody, PM fraction control $\mathrm{Na}+/ \mathrm{K}+$ ATPase (absent in cytosol) by anti- $\alpha 1 \mathrm{Na}+/ \mathrm{K}+$ ATPase antibody and cytosol fraction control Tubulin by anti- $\alpha-$ Tubulin antibody. ${ }^{\star} p \leq 0.05 ;{ }^{\star *} p \leq 0.01$.

\section{A}
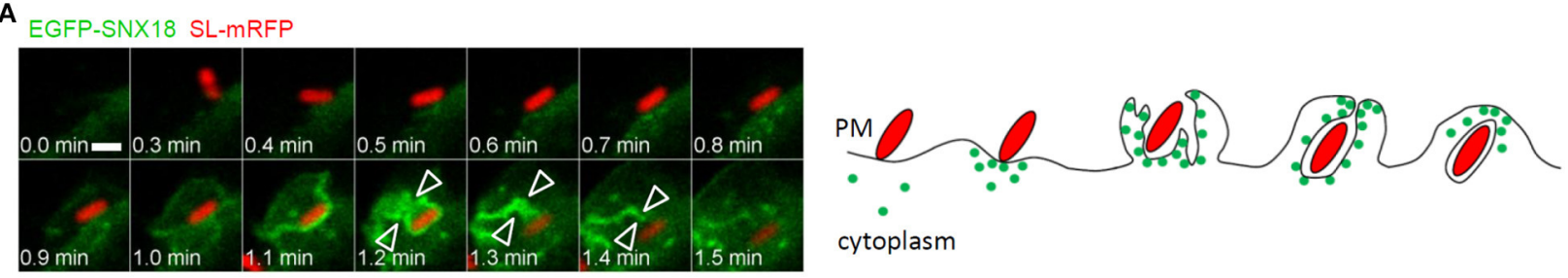

B
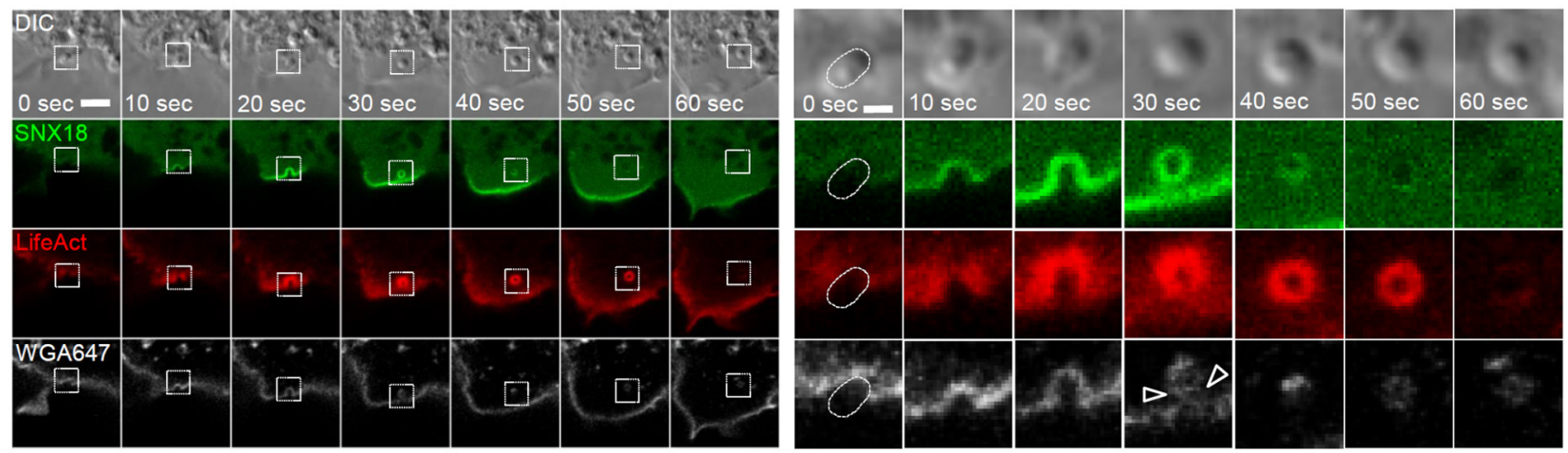

FIGURE 3 | The recruitment of SNX18 to the site of SCV formation correlates with a burst of actin polymerization and membrane scission: (A) Series of confocal sections from a time-lapse of EGFP-SNX18-expressing cells infected with SL-mRFP. SNX18 relocates from cytosol to Salmonella-induced membrane ruffles at the site of bacterial attachment with a transient accumulation of SNX18 during the SCV scission from the plasma membrane (arrowheads). Bar $=2 \mu \mathrm{m}$. The diagram illustrates SNX18 (green dots) localization during bacterial internalization and at the early stage of the SCV formation. (B) Series of confocal sections from a time-lapse of HEK293 cells coexpressing EGFP-SNX18 with LifeAct-Ruby and infected with SL-mRFP in the presence of wheat germ agglutinin coupled with Alexa Fluor 647 (WGA-647). The burst of SNX18 and LifeAct-Ruby probe during bacteria internalization and SCV scission (indicated by arrowheads) and their depletion after the SCV detachment from the plasma membrane. Entering bacteria are visible in DIC. The panel on right shows enlarged details from the left panel. Bars $=10 \mu \mathrm{m}$ (left panel) or $2 \mu \mathrm{m}$ (right panel).

similar extent as the full length SNX18, its co-localization with Dynamin-2 and N-WASP was markedly reduced as shown on quantification of the co-localization within bacteria-containing membrane ruffles (Figures 4A,B).

Together, these results provide the evidence that $S$. Typhimurium triggers rapid recruitment of cytosolic SNX18 to the site of invasion to function in the actin-driven formation and scission of the nascent SCV from the plasma membrane together with Dynamin-2 and N-WASP. We propose that the SH3 domain is dispensable for SNX18 targeting to the plasma membrane but is required for recruitment of Dynamin-2 and $\mathrm{N}$-WASP to distinct membrane subdomains of membrane ruffles during formation and closure of the nascent SCV.

\section{Mutation of SNX18 Impairs S. Typhimurium Internalization}

The above results suggested that the SNX18 can mediate the process of $S$. Typhimurium internalization into host cells by acting as a scaffold for the recruitment of Dynamin-2 and $\mathrm{N}$ WASP via its SH3 domain. Therefore, we next investigated whether transient overexpression of SNX18 mutants lacking the SH3 domain (SNX18 $\triangle \mathrm{SH} 3$ ) or phosphoinositide binding mutant (SNX18:R303Q) will affect the overall efficiency of bacteria internalization. The design of the phosphoinositide binding mutant was based on a structural study of SNX9 (a paralog of SNX18), which demonstrated that mutation of the corresponding arginine (Arg286) within $\alpha 1$ loop of phosphoinositide-binding pocket abrogates SNX9 binding to liposomes (Pylypenko et al., 2007). HEK293 cells were transfected with plasmids encoding wild-type or mutant SNX18 and infected with mCherryexpressing $S$. Typhimurium. The amount of internalized bacteria was determined by using a quantitative immunofluorescence assay. At $10 \mathrm{~min}$ post infection, cells overexpressing wild-type SNX18 exhibited a significantly higher number of internalized bacteria when compared with control cells. In contrast, a decrease in bacterial uptake was found in cells transfected with SNX18: $\triangle \mathrm{SH} 3$ or SNX18:R303Q constructs relative to 
A
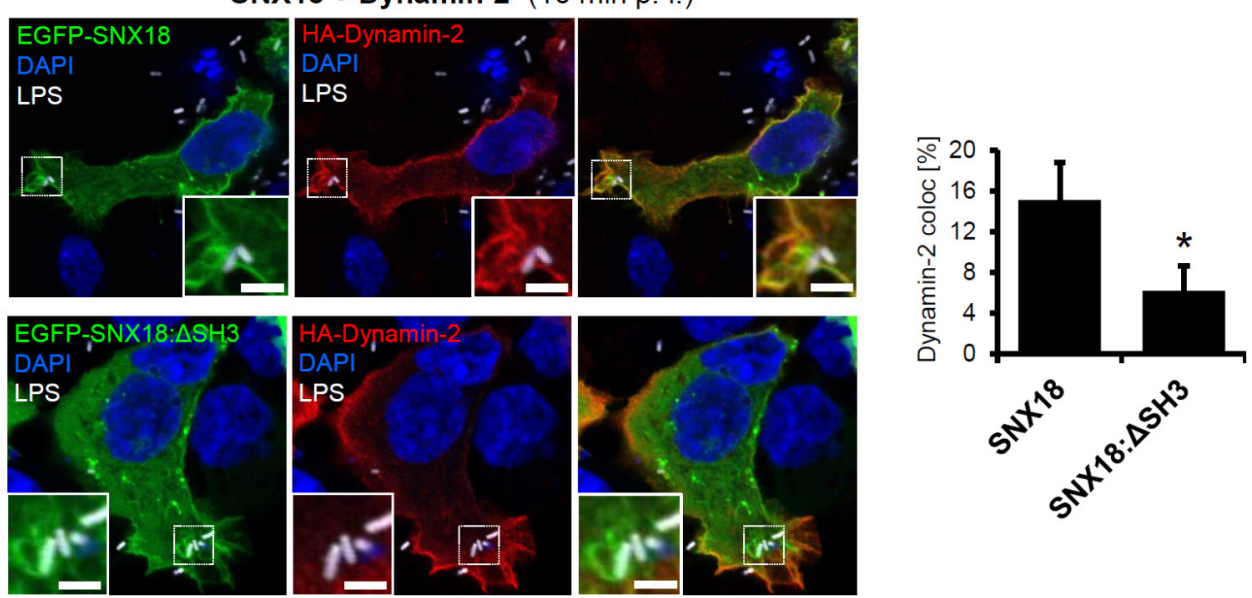

B

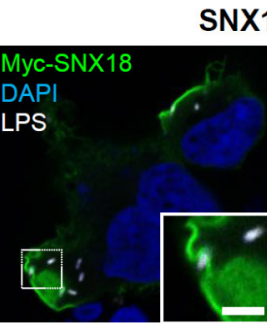

SNX18 + N-WASP (10 min p. i.)
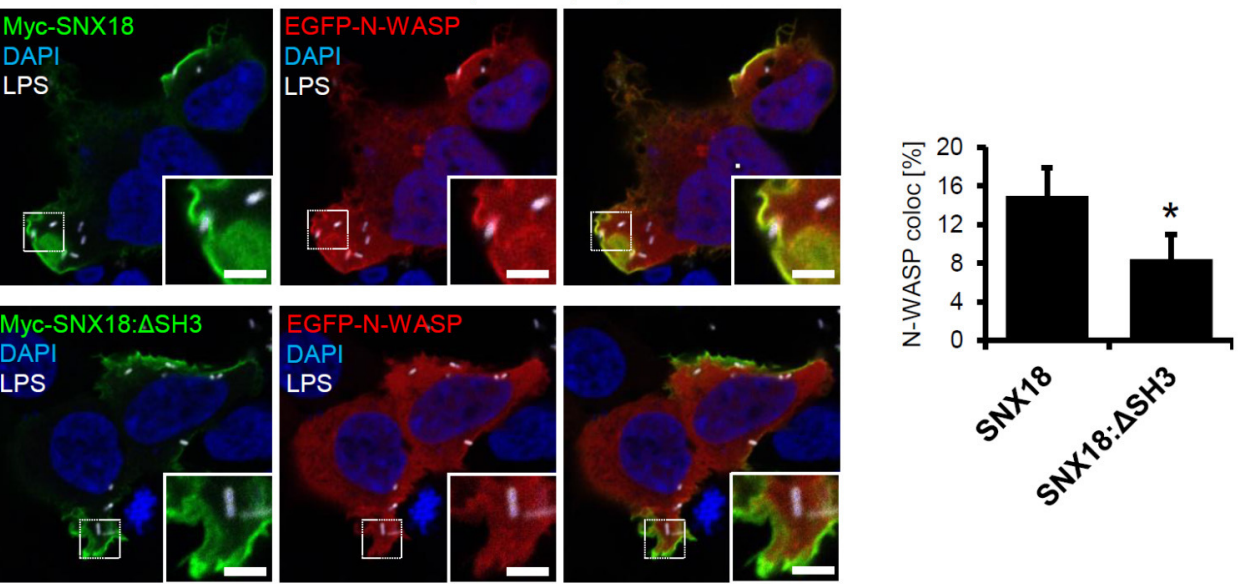

FIGURE 4 | SNX18 functions as a scaffold for N-WASP and Dynamin-2 recruitment to the site of SCV formation. Co-localization between SNX18 and Dynamin-2 (A) or SNX18 and N-WASP (B) within Salmonella-containing membrane ruffles is significantly reduced in cells expressing EGFP-SNX18 construct lacking the SH3 domain. Cells co-expressing EGFP- or Myc-tagged SNX18 with HA-tagged Dynamin2 (A) or EGFP-N-WASP (B) fixed 10 min post infection (p. i.) and immunolabeled with anti-myc or anti-HA antibody followed by secondary antibody coupled to Alexa Fluor 546. Bacteria were detected by anti-LPS antibody followed by Alexa Fluor 405 secondary antibody and DNA was stained with DAPI. The fluorescent images are pseudocolored as indicated. Bars $=5 \mu \mathrm{m}$. Co-localization of indicated proteins in bacteria-containing membrane ruffles was quantified. The ROls were limited to bacteria-containing membrane ruffles (enlarged inserts) cropped to identical square size $(12 \times 12 \mu \mathrm{m})$. Co-localization is presented as a percentage of voxels which have both channel 1 and channel 2 intensities above threshold, expressed as a percentage of the total number of pixels in the image (including zero-zero pixels); $t$-test, ${ }^{*} p<0.05$; Between 10-20 ROI per sample were analyzed and bars indicate the mean + standard errors within typical experiment.

the control cells (Figure 5A). These results demonstrated that the efficiency of bacteria internalization is affected by loss-offunction mutations in SNX18.

To determine whether overexpression of SNX18: $\triangle \mathrm{SH} 3$ and SNX18:R303Q mutants reduces bacteria internalization by interfering with the SCV formation, we analyzed the kinetics of this process by live cell imaging. The SNX18: $\triangle \mathrm{SH} 3$ exhibited little difference in sub-cellular localization, recruitment to the site of bacterial internalization and transient association with the nascent SCV when compared to the full length SNX18 (Figure 4). In contrast, the phosphoinositide-binding mutant SNX18:R303Q remained exclusively cytosolic and exhibited no change during bacterial internalization. Although the internalization of bacteria was not blocked in cells expressing the SNX18 mutants, we frequently observed a delay between docking of the bacteria on the host cell and completion of the internalization process (scission of the SCV from the plasma membrane) in comparison to cells expressing the full-length SNX18 construct (Figure 5B and Movies 3-5).

Taken together, these data suggest that transient overexpression of SNX18: $\triangle \mathrm{SH} 3$ or SNX18:R303Q may reduce the overall efficiency of $S$. Typhimurium internalization at the onset of infection, likely through defects or a delay in the recruitment of proteins involved in formation 
A

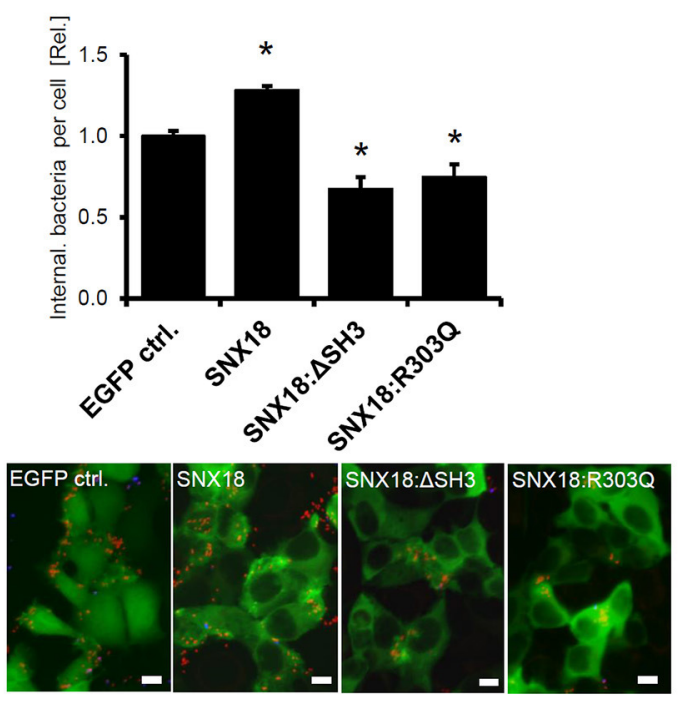

B
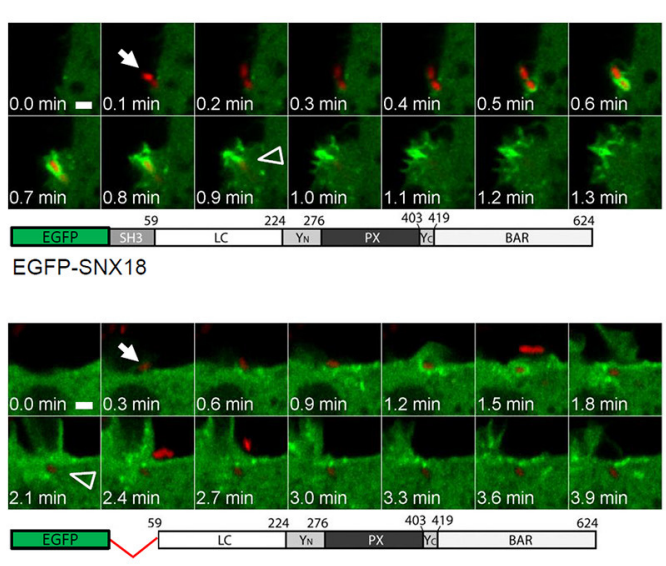

EGFP-SNX18: $\triangle \mathrm{SH} 3$

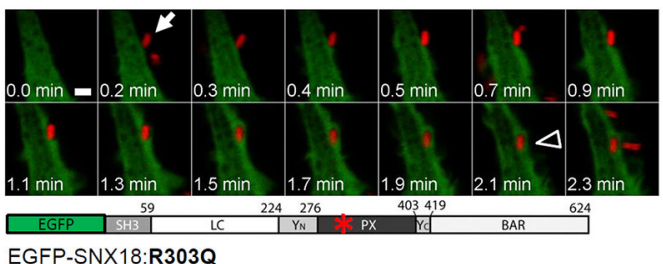

FIGURE 5 | SNX18 requires the SH3 domain and an intact Ptdlns-binding site to facilitate the uptake of the bacteria. (A) Quantification of bacteria internalization in cells overexpressing full length SNX18, SNX18 lacking the SH3 domain (SNX18: $\triangle \mathrm{SH} 3$ ) or phosphoinositide binding mutant SNX18:R303Q in comparison to control cells (expressing EGFP only). Cells were fixed at $10 \mathrm{~min}$ post infection with SL-mRFP and intracellular bacteria were quantified by image analysis with exclusion of extracellular bacteria (immunolabeled with anti-LPS antibody). The average values in control cells were normalized to one to demonstrate the fold change in cells overexpressing SNX18 or the SNX18 mutants. ${ }^{*} p<0.05$. Bars indicate the mean + standard deviation between three experiments. Below: Examples of immunofluorescence images used for quantification. Bars $=10 \mu \mathrm{m}$. (B) Live imaging of HEK293 cells overexpressing EGFP-tagged SNX18, SNX18: $\triangle$ SH3, or SNX18:R303Q constructs and infected with SL-mRFP. Note the delay between bacteria first contact with the cell (arrows) and complete internalization (arrowheads) in cells expressing both SNX18 mutants and no sign of SNX18:R303Q recruitment to the site of bacteria invasion. Series of confocal sections from representative time-lapse are shown. Schematic diagrams of each construct are shown below. Bars $=2 \mu \mathrm{m}$.

and/or scission of the nascent SCV. Because the uptake of bacteria in cells overexpressing the SNX18 mutants was only partially reduced, as we observed for the SNX18 knockdown cells, we propose that the SNX18-mediated pathway may represent one of the two independent but complementary invasion mechanisms recently described for S. Typhimurium (Hanisch et al., 2011) that are regulated either by SopB or by SopE/SopE2 effectors of SPI1-T3SS.

\section{Recruitment of SNX18 Is Triggered by Salmonella Ptdlns-Phosphatase SopB}

Internalization of $S$. Typhimurium is mediated by orchestrated action of several SPI1-T3SS effectors, including SopB, SopD, SopE, SopE2, SptP, SipA, and SipC that disrupt tight junctions and cell polarity (Boyle et al., 2006), induce actin cytoskeleton remodeling (Cain et al., 2008) and promote SCV formation (Terebiznik et al., 2002; Hernandez et al., 2004; Bakowski et al., 2007). We therefore reasoned that the recruitment of SNX18 to the sites of bacterial invasion may be triggered by one or more of these effectors.

First we analyzed whether SNX18 mobilization in infected cells is dependent on activity of SPI-T3SS and independent of
SPI2-T3SS. The SPI1-T3SS deficient mutant of S. Typhimurium, $\triangle i n v A$, is non-invasive and its contact with the EGFP-SNX18 expressing cells also failed to induce SNX18 relocation to the site of bacterial attachment at the cell surface. In turn, when cells were infected with the SPI2-T3SS deficient mutant, $\Delta s s a R$, a burst of SNX18 was detected at the site of bacteria internalization to the extent similar to cells infected with the wild type bacteria (Figure 6A). To examine whether a particular SPI1-T3SS effector is sufficient to trigger SNX18 recruitment, we used transient ectopic expression of myc-tagged fusion proteins of individual SPI1-T3SS effectors in cells with stable expression of EGFPSNX18. To minimize potential toxicity of effectors on the cell structure and functions, the cells were fixed and analyzed early at $4-6 \mathrm{~h}$ post transfection when the proteins were become detectable by indirect immunofluorescence. Our screen revealed that SNX18 retained its cytosolic localization except when coexpressed with SopB, where a substantial fraction of SNX18 accumulated in plasma membrane (Figure 6B). Although SNX18 remained cytosolic when co-expressed with the other SPI1 effectors, we did observe extensive membrane ruffles in SopE2expressing cells and impaired actin cytoskeleton organization in cells expressing SipA, SipC, and SptP, which is consistent with the function of these effectors previously reported in the 
A

SL $\triangle$ invA

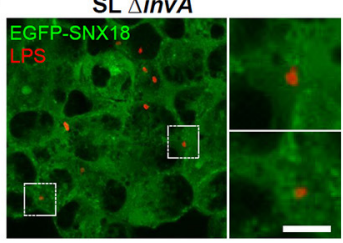

SL $\triangle S s a R$

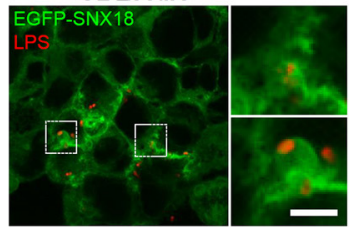

B
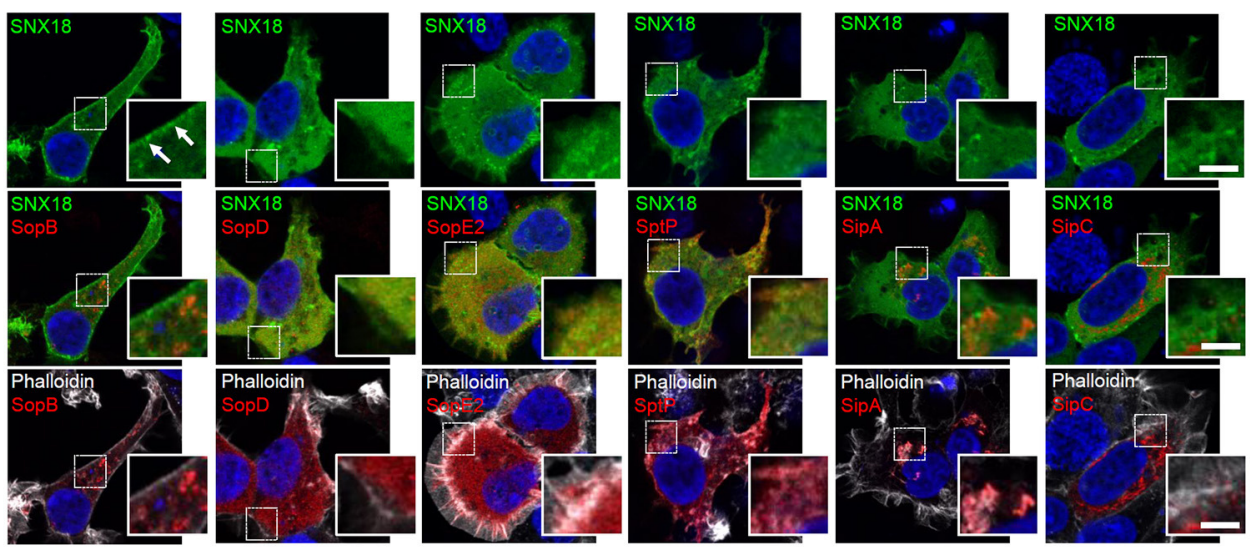

FIGURE 6 | Activity of SPI1-T3SS effector SopB is necessary and sufficient for SNX18 recruitment to the plasma membrane. (A) SNX18 recruitment to the site of bacterial internalization is markedly perturbed in cells infected with the SPI1-T3SS inactive mutant $\triangle i n v A$ (left) while in cells infected with the SPI2-T3SS inactive mutant $\Delta s s a R$ (right) the burst of SNX18 at the site of bacterial internalization is comparable to that of induced by the wild type bacteria. Bacteria were detected by indirect immunofluorescence using anti-LPS antibody followed by secondary antibody coupled to Alexa Fluor 546 . Bars $=5 \mu \mathrm{m}$. (B) Cells with stable expression of EGFP-SNX18 and transient ( 6 h) ectopic expression of myc-tagged SPI1-T3SS effectors with reported function in S. Typhimurium internalization. After fixation, the effectors were detected by anti-myc antibody followed by secondary antibody coupled to Alexa Fluor 546 (red), DNA was labeled with DAPI (blue), and F-actin was labeled by Phalloidin Alexa Fluor 647 (pseudocolored in white); Only expression of SopB resulted in depletion of cytosolic SNX18 and accumulation of SNX18 in the plasma membrane. Bars $=5 \mu \mathrm{m}$.

literature (Pizarro-Cerda and Cossart, 2004; Haglund and Welch, 2011).

To confirm that SopB is essential and sufficient for SNX18 recruitment to the sites of bacterial invasion, we next infected cells with an isogenic $\operatorname{sop} B$ mutant of $S$. Typhimurium $(\triangle \operatorname{sop} B$ mutant). In comparison to the wild type $S$. Typhimurium, the $\triangle$ sopB mutant failed to recruit cytosolic SNX18 to the plasma membrane and to the site of bacterial internalization (Figure 7A). Moreover, quantification of bacterial internalization revealed that the reduction in numbers of internalized wild type S. Typhimurium (SLwt) in SNX18 knockdown cells can be restored by transient overexpression of myc-tagged SNX18. In contrast, numbers of internalized mutant (SL $\Delta s o p B)$ were not significantly changed in SNX18 knockdown cells relative to control knockdown cells (Figure 7B). Together, these results further confirm that SNX18 functions in SopB-mediated internalization pathway of bacteria.

We next aimed to determine whether SNX18 recruitment was dependent on the ability of SopB to modify phosphoinositides. We constructed myc-tagged mutants of SopB that have been shown to abolish the phosphoinositide phosphatase activity of this effector: C460S, R466A, and K528A (Drecktrah et al., 2004). In cells co-expressing EGFP-SNX18 with wild type SopB, a substantial amount of SNX18 localized to the plasma membrane, while SopB localized primarily to enlarged endosomal structures most likely induced as a result of PtdIns(3)P accumulation at their membrane (Figure 7C). In contrast, all three SopB mutant proteins localized to the plasma membrane and small peripheral endosomal structures, suggesting they still can associate with the plasma membrane but fail to induce accumulation of enlarged PtdIns(3)P-positive endosomes. The co-expression of SopB mutant proteins did not alter the predominantly cytosolic localization of SNX18 and neither wild type mycSopB nor the myc-tagged sopB mutant proteins co-localized with SNX18. Importantly, the failure of $\triangle \operatorname{sop} B$ mutant bacteria to trigger SNX18 relocation to the membrane ruffles was fully restored when $\operatorname{sop} B$ was expressed from a plasmid in strain $\mathrm{SL} \Delta s o p B+\mathrm{pSopB}$. However, complementation was not achieved with introduction of plasmids encoding the inositolphosphatasedeficient mutant of SopB (C460S) (Figure 7D). Furthermore, SopB activates Akt/protein kinase B in epithelial cells infected by $S$. Typhimurium (Steele-Mortimer et al., 2000) and lack of Akt activation in cells infected with $\triangle s o p B$ mutant was efficiently restored in cells infected with SL $\Delta s o p B+\mathrm{pSopB}$ strain (data not shown) what implies that the plasmid-encoded SopB in SL $\Delta$ sopB was efficiently translocated to infected cells.

Since SopB can also indirectly enhance Rho GTPase activation (Patel and Galan, 2006), we next examined whether SNX18 

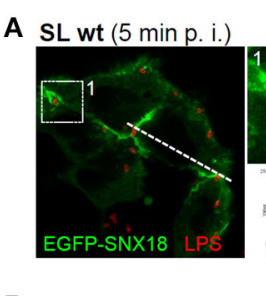

B

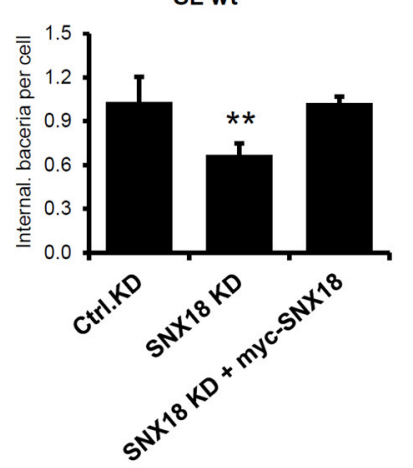

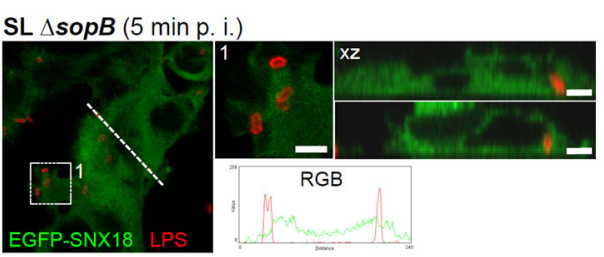

SL $\triangle$ sopB

C

\section{SopB wt}

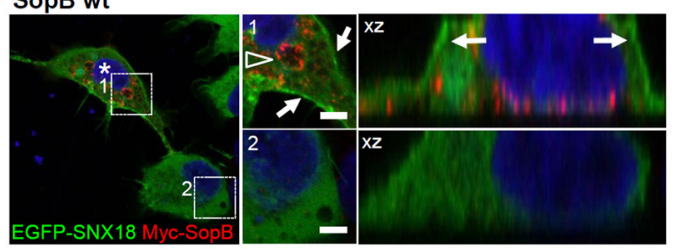

SopB:C460S
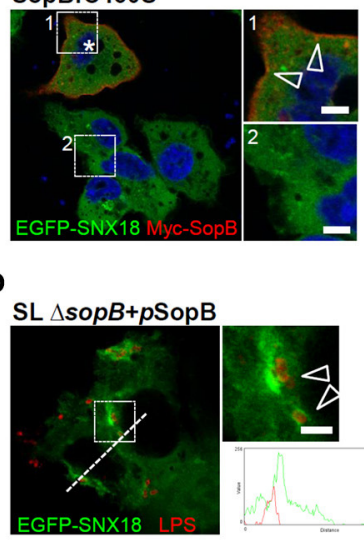

SopB:R466A

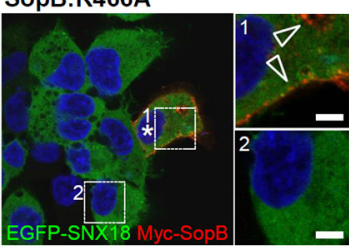

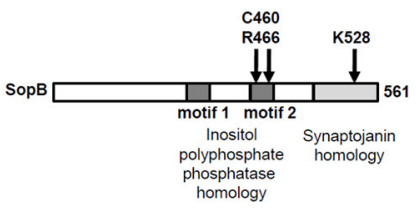

SopB:K528A

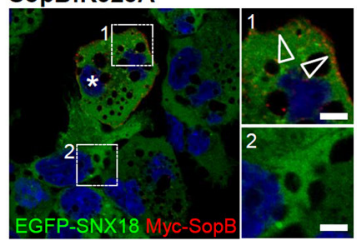

FIGURE 7 | The SNX18-mediated invasion pathway of S. Typhimurium relies on inositol-phosphatase activity of SopB (A) S. Typhimurium mutant $\triangle$ sopB failed to trigger SNX18 recruitment to the site of invasion. EGFP-SNX18 expressing cells infected with wild type (left) or $\Delta$ sopB bacteria (right). Cells were fixed 10 min post infection (p. i.) and bacteria were labeled with anti-LPS antibody followed by secondary antibody coupled to Alexa Fluor 546 (red). The detail of Salmonella-containing ruffle (1) and the projection of confocal z-stack (xz) is shown together with the fluorescence intensity line profile (RGB) across the cell. Bars $=5 \mu \mathrm{m}$. (B) Quantification of the bacterial internalization (wild type and $\triangle S O p B$ ) in control knockdown cells, SNX18 knockdown cells and SNX18 knockdown cells transfected with myc-SNX18. Extracellular bacteria were detected by anti-LPS antibody followed by secondary antibody coupled with Alexa Fluor 405 and myc-SNX18 was detected by anti-myc antibody followed by secondary antibody coupled with Alexa Fluor 647. Numbers of internalized bacteria (mRFP-positive and LPS-negative) were counted by image analysis and average numbers per cell are shown. Bars indicate the mean + standard deviation between three experiments. (C) Localization of EGFP-SNX18 after ectopic expression of SopB or Ptdlns-phosphatase defective mutants of SopB in non-infected cells. The wild type SopB localizes to endosomes and enlarged vacuoles (arrowheads) and induces SNX18 accumulation at the plasma membrane (arrows) whereas SopB with point mutation within the inositol polyphosphate 4-phosphatase homology (IPPH) motif 2 (C460S or R466A) or within the synaptojanin homology domain (K528A) localizes to the plasma membrane (arrowheads) and peripheral vesicles without visible changes to SNX18 localization. Cells were fixed $6 \mathrm{~h}$ post transfection, labeled with DAPI and myc-tagged SopB was detected by anti-myc antibody followed by secondary antibody coupled to Alexa Fluor 546 (red). Bars $=5 \mu \mathrm{m}$. (D) The failure of $\Delta s$ sopB mutant bacteria to trigger SNX18 recruitment during internalization can be rescued by complementation using the full length SopB but not the inositol phosphatase deficient SopB:C460S. The HEK293 cells expressing EGFP-SNX18 were infected with the $\triangle \operatorname{sop} B$ mutant bacteria transformed with pWSK29 plasmid encoding $\operatorname{sop} B$ or $\operatorname{sop} B: C 460 S$. Immunofluorescence detection of bacteria and the RGB profiles were performed as in (A), Bars $=5 \mu \mathrm{m} .{ }^{\star \star} p \leq 0.01$. 
recruitment to the plasma membrane occurs independently or as a consequence of Cdc42 and/or Rac1 activation. Upon expression of constitutively active (CA) forms of Racl or Cdc42 in noninfected cells, Rac1, and Cdc42 localized to the plasma membrane and Racl induced formation of extensive membrane ruffles. However, in both cases SNX18 retained its cytosolic localization. Expression of dominant negative (GTPase-defective) forms of Cdc42 and Rac1 did not inhibit SNX18 recruitment to the site of bacterial invasion (Figure S2) and inhibition of Rac1-GTPase activity by specific, cell-permeable and reversible inhibitor NSC23766 did not perturb SNX18 recruitment to the site of bacteria internalization (Figure S3).

Collectively, these results demonstrate that relocation of SNX18 to Salmonella-induced membrane ruffles occurs independently of $\mathrm{Cdc} 42$ and Rac1 activation and that the PtdIns-phosphatase activity of SopB is necessary to drive SNX18 recruitment to the site of bacteria invasion. However, SNX18 and SopB probably do not directly interact, in agreement with our co-immunoprecipitation experiments detecting no interaction between SNX18 and ectopically expressed SopB (not shown).

\section{SNX18 Recruits to Membrane Subdomains Enriched in Ptdlns(3,4)P2}

Given that the phosphoinositide binding of SNX18 is essential for membrane recruitment, and this recruitment is dependent on the PtdIns-phosphatase activity of SopB, we next investigated whether SNX18 is recruited to the plasma membrane subdomains in response to SopB-mediated manipulation of phosphoinositide composition, since it has been previously reported that SopB is essential for local increase in PtdIns(3,4)P2 and PtdIns(3,4,5)P3 and depletion of PtdIns(4,5)P2 at Salmonella-induced membrane ruffles (Terebiznik et al., 2002; Mason et al., 2007; Mallo et al., 2008).

To visualize the local enrichment or depletion of particular membrane-bound phosphoinositides at the site of bacterial invasion, EGFP-tagged fusion proteins with (i) FERM domain of Ezrin, (ii) Pleckstrin Homology $(\mathrm{PH})$ domain of Akt (also known as Protein Kinase B), or (iii) 2xFYVE domain of mouse Hours protein (hepatic growth factor-regulated tyrosine kinase substrate) were expressed in HEK293 cells. The FERMEGFP recognizes and binds to PtdIns(4,5)P2 while EGFPAKT-PH has affinity to PtdIns(3,4)P2 and/or PtdIns(3,4,5)P3 and $2 \mathrm{xFYVE-EGFP}$ has been used as a highly selective probe of PtdIns(3)P (James et al., 1996; Barret et al., 2000; Pattni et al., 2001). Following infection of these cells with mRFPexpressing bacteria, the FERM-EGFP with exclusive plasma membrane localization became markedly enriched at the cell periphery likely in response to an increase in membrane ruffling triggered by invading bacteria. However, closer inspection of membrane ruffles revealed a substantial local depletion of FERM-EGFP at the sites of bacterial internalization within these membrane subdomains and no association of FERMEGFP was detected on fully internalized SCV (Figure S4). The EGFP-AKT-PH construct localized preferentially to the plasma membrane and to Salmonella-induced ruffles and exhibited substantial increase in fluorescence density around bacteria during the SCV formation but not around fully internalized SCV (Figure S4). In cells expressing the 2xFYVE-EGFP, the construct localized to tubular endosomal structures, exhibited a limited recruitment to the site of bacterial invasion, but associated markedly with fully internalized SCV (Figure S4). These results demonstrate that a transition occurs between accumulation of PtdIns(3,4)P2/PtdIns(3,4,5)P3 and PtdIns(3)P on the membrane of the SCV during early steps of the organelle biogenesis, which is in agreement with previous reports (Pattni et al., 2001; Mallo et al., 2008).

To determine whether SNX18 recruits simultaneously to the same membrane subdomains as EGFP-AKT-PH construct, we monitored the dynamics of EGFP-AKT-PH and mCherrySNX18 recruitment in live cells during the $S$. Typhimurium internalization. A substantial amount of EGFP-AKT-PH localized to the plasma membrane prior to infection, yet a distinct increase in EGFP fluorescence intensity was detected at the site of bacterial internalization. Moreover, kinetics of relative increase in fluorescence intensity indicated that Akt-PH and SNX18 recruited simultaneously to bacteriainduced membrane ruffles within the first $30 \mathrm{~s}$ of bacteria internalization (Figure 8A and Movie 6). Since the EGFP-AKT$\mathrm{PH}$ probe does not distinguish between Ptdins $(3,4) \mathrm{P} 2$ and PtdIns $(3,4,5) \mathrm{P} 3$, we next aimed to determine whether SNX18 exhibits selective affinity to one of these phosphoinositides. For this, we co-expressed mCherry-SNX18 with EGFP-tagged PH domain of Tandem PH-domain containing protein (TAPP1) or with EGFP-tagged $\mathrm{PH}$ domain of Bruton's tyrosine kinase (Btk). The PH domain of TAPP1 possesses specific affinity to PtdIns(3,4)P2 (Dowler et al., 2000), while the PH domain of Btk binds exclusively to PtdIns(3,4,5)P3 (Salim et al., 1996). The SNX18 and TAPP1-PH became simultaneously enriched at the site of bacterial docking and internalization and a substantial increase in co-localization was detected during this process (Figure 8B and Movie 6). In contrast, we detected little increase in Btk-PH at the site of bacterial invasion and co-localization with SNX18 was below detection level (Figure 8C and Movie 6). Moreover, in cells overexpressing SNX18 together with AKT-PH or TAPP1-PH constructs the burst of SNX18 recruitment at the plasma membrane triggered by invading bacteria was notably attenuated in comparison to cells expressing mCherry-SNX18 alone or together with the Btk-PH-EGFP. These data suggest that SNX18, TAPP1-PH and AKT-PH can compete for binding to PtdIns(3,4)P2 and that SNX18 is recruited to membrane subdomains selectively enriched in PtdIns(3,4)P2.

Since we observed the simultaneous burst of SNX18 and Akt within Salmonella-induced membrane ruffles, we were interested whether SNX18 recruitment is dependent on the activation of Akt, which can also be triggered by the inositol phosphatase activity of SopB (Steele-Mortimer et al., 2000). Pre-treatment of cells with Akt-specific inhibitor AKTil/2 did not markedly perturb recruitment of EGFP-SNX18 to the site of bacteria invasion, while the phosphorylation of Akt elicited by wild type $S$. Typhimurium (but less by $\triangle \operatorname{sop} B$ mutant) 

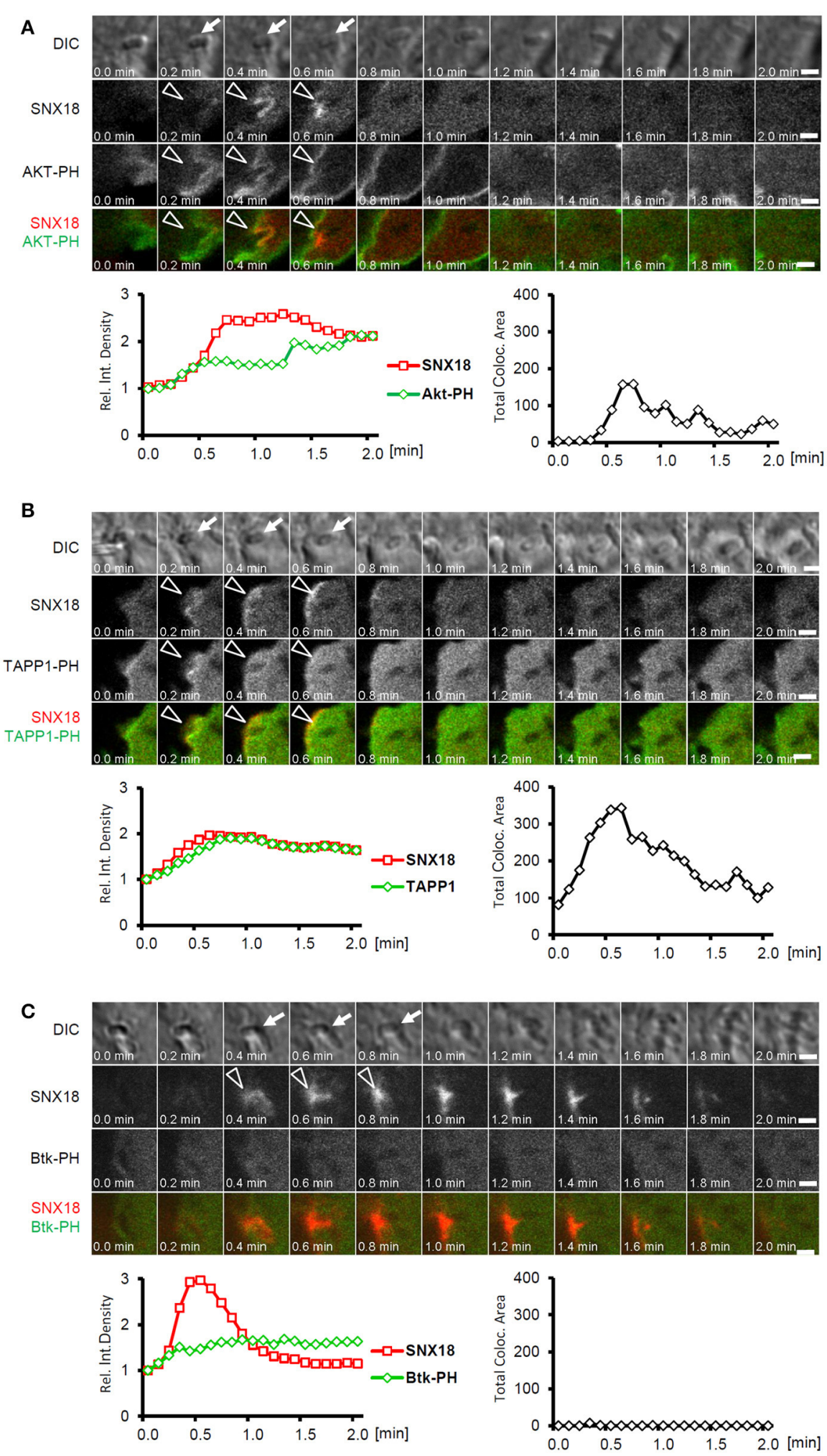

FIGURE 8 | SNX18 is recruited to Ptdlns(3,4)P2-enriched membrane subdomains. Correlation between recruitment kinetics of SNX18 and that of Akt-PH (A), TAPP1-PH (B), and Btk-PH (C) during bacteria internalization; Panels show series of still images from time-lapse movie of cells co-transfected with mCherry-SNX18 and Akt-PH-EGFP (A), TAPP1-EGFP (B), and Btk-PH-EGFP (C) and infected with non-fluorescent bacteria (indicated by arrows in DIC channel); Simultaneous recruitment of SNX18 and Akt-PH or TAPP1 to the site of bacterial internalization is indicated by arrowheads; Below: Correlation of transient increase in fluorescent density between mCherry and EGFP (left) were analyzed from sequence of 21 images (values at $T=0$ were normalized to 1 to show a relative increase/decrease over time); Time profile of total co-localization area between mCherry and EGFP signal (right); Bars $=5 \mu \mathrm{m}$. Shown are results from a typical live imaging experiment. 
at $10 \mathrm{~min}$ post infection was completely abolished (data not shown).

Taken together, these results demonstrate that SNX18 is recruited to Salmonella-induced membrane invaginations in response to SopB-mediated accumulation of $\operatorname{PtdIns}(3,4) \mathrm{P} 2$ and that SNX18 recruitment occurs together with Akt but independently of Akt activation.

\section{DISCUSSION}

$S$. Typhimurium orchestrates its internalization into host cells through the action of multiple SPI1-T3SS effectors, which hijack the host macropinocytosis pathway for bacterial uptake. A critical step in this pathway is the formation and scission of the nascent SCV mediated by dynamin-2 and N-WASP, however the precise mechanism of the recruitment of these proteins to the sites of bacterial invasion has not been described. Here we identify a function for SNX18 in this process and demonstrate that engagement of SNX18 occurs via the action of the Salmonella SPI1-T3SS effector SopB. The recruitment of SNX18 to the plasma membrane is induced by the expression of SopB alone and SopB's phosphatidylinositol phosphatase activity is essential for the spatial and temporal recruitment of SNX18 induced during S. Typhimurium invasion. Furthermore, mutation of the phosphoinositide binding site within the PX domain of SNX18 completely abolished SNX18 recruitment to the plasma membrane. We propose that activated SNX18 binds to phosphoinositides locally modified during Salmonella invasion and functions as a membrane scaffold for the recruitment of the molecular components of actin-driven membrane remodeling. This study identified SNX18 as a novel target of $S$. Typhimurium and we propose that in contrast to other sorting nexins that have been previously linked to the SCV maturation, SNX18 is engaged at the onset of bacterial internalization to function in biogenesis of membrane ruffles and macropinocytosis. Hereby S. Typhimurium recruits SNX18 and its associated proteins via inositol-phosphatase activity of SopB to locally and transiently upregulate macropinocytosis and thus facilitate invasion of the host cell. Nevertheless, the invasion process is-in parallel-orchestrated and dependent on additional Salmonella effector proteins including SopE and SopE2 which allows $S$. Typhimurium to fine-tune the efficiency of invasion into various cell types and/or via multiple endocytic pathways. Like SNX18, SNX9 is recruited to the site of Salmonella invasion, via its PX domain, and that the capacity of Salmonella to invade cells with a transient siRNA knock-down of SNX9 is reduced (Piscatelli et al., 2016). Therefore, it appears that SNX18 and SNX9 may have redundant functions during Salmonella invasion.

Studies into Salmonella internalization have been complicated by an array of mechanisms these bacteria have evolved to invade macrophages and polarized or non-polarized epithelial cells, and to activate or suppress various host cell signaling pathways. Thus, blocking of a single pathway often results in insignificant changes in the ability of Salmonella to invade cells. So far, only cytochalasin D has been found to completely block $S$. Typhimurium internalization in both epithelial cells and macrophages (Finlay et al., 1991; Forsberg et al., 2003), highlighting the indispensable role of actin cytoskeleton remodeling for bacteria entry. Moreover, two independent but complementary mechanisms of SopE-dependent and SopBmediated internalization pathways of $S$. Typhimurium have been described (Stender et al., 2000; Hanisch et al., 2011). The first is characterized by the activation of Rac1/Cdc42 followed by activation of WAVE and WASH complex and Arp2/3mediated actin polymerization, leading to extensive membrane ruffle formation, while the second is mediated by Rho kinase and Myosin II activity and occurs through plasma membrane invaginations without membrane ruffling (Stender et al., 2000; Hanisch et al., 2011). Indeed, we observed that bacteria entered the cell by two morphologically distinct mechanisms, but how these mechanisms differ at the molecular level is difficult to discern because both pathways seems to be utilized when multiple bacteria infect a single cell.

We found that cells overexpressing SNX18 exhibited a considerably increased rate of macropinocytosis, whereas the impact on $S$. Typhimurium internalization was relatively mild. Similarly, in SNX18 knockdown cells, the rate of macropinocytosis was reduced to a greater extent than internalization of bacteria. We argue that since $S$. Typhimurium infection elevates macropinocytosis via the action of translocated effectors, any additional gain from the direct expression of SNX18 might be limited. The fact that we observed only partial reduction in bacterial internalization efficiency in SNX18 knockdown cells may reflect the ability of bacteria to trigger the internalization process via a complementary mechanism which can be independent of SopB and SNX18. It will be interesting to find whether $S$. Typhimurium utilizes multiple pathways simultaneously or possesses an ability to fine-tune the invasion efficiency according to environmental cues. Nevertheless, the reduced invasiveness of SopB-deficient mutant of S. Typhimurium strain SL1344 or that of the wild type of SopE-deficient strain ST12023, both reported in the literature (Bakowski et al., 2007; Clark et al., 2011), suggests that the ability of these bacteria to trigger and utilize multiple internalization mechanisms enhances the net invasion efficiency. Thus, although we do not question that $S$. Typhimurium can utilize complementary pathway(s), our study describes the role of SNX18 in SopB-dependent invasion mechanism which significantly facilitates bacterial internalization.

The cells overexpressing the SNX18 mutant $\Delta$ SH3 or R303Q exhibited a mild but significant decrease in bacterial invasion yet the mechanism by which they interfere is unclear. The most likely explanation is that upon recruitment to the plasma membrane, the SNX18: $\triangle \mathrm{SH} 3$ can interact with the endogenous pool of SNX18 resulting in production of a higher amount of SNX18 dimers with a reduced functionality. In contrast, similar pseudo-dimer formation between SNX18:R303Q and endogenous SNX18 would induce partial cytosolic retention of SNX18. Indeed, we did not detect any increase in fluorescence 
intensity of SNX18:R303Q at the site of bacterial internalization or at any other membrane subdomains. Nevertheless, Sorting nexin 9,18 , and 33 can readily be found as homodimers when isolated from cell lysates. Formation of heterodimers between SNX9-SNX18 and SNX9-SNX33 in vitro has been previously reported (Zhang et al., 2009; Park et al., 2010), but not confirmed by a recent study (van Weering et al., 2012) and ourselves (data not shown), and whether possible heterodimers represent functional units in intact cells in vivo still remains to be determined.

Interaction between SNX18 and membrane-bound phosphoinositides is essential for regulation of SNX18 function, yet the phosphoinositide specificity of SNX18 PX domain remains unclear. Filter binding assays and liposome co-sedimentation experiments using a GST fusion of SNX18 PX domain or PX-BAR module identified affinity for PtdIns(3,4)P2, PtdIns(3,5)P2, and PtdIns(4,5)P2 (Haberg et al., 2008; Nakazawa et al., 2011) and similar broad lipid specificity has also been reported for SNX9 (Shin et al., 2008; Yarar et al., 2008). However, the specificity of SNX18 and/or SNX9 for individual phosphoinositides in live cells is unknown. Using live cell imaging, our study provides insight into the recruitment of SNX18 to membrane subdomains enriched in PtdIns(3,4)P2 and highlights the importance of cell context in this assay because the spatio-temporal aspects of recruitment or depletion of phosphoinositide binding proteins is difficult to characterize by biochemical approaches. It is important to note that even transient overexpression of phosphoinositide sensing probes may imbalance the phosphoinositide metabolism by irreversible binding of these probes to particular phosphoinositide subspecies. This, as our results suggest, would also lead to affinity-dependent competition between SNX18 and the probes for binding PtdIns(3,4)P2. Nevertheless, our conclusions were based on comparison of recruitment kinetics between SNX18 and individual PtdIns-probes and the kinetics seemed to be comparable to that of cells expressing SNX18 without the PtdIns-probes.

Identification of SNX18 as a host cell factor involved in SopB-mediated formation of the nascent SCV at the plasma membrane builds upon the previously described role of SopB (formerly SigD) in hydrolysis of PtdIns(4,5)P2 during this process (Terebiznik et al., 2002). Terebiznik et al. (2002), demonstrated that the break-down of PtdIns(4,5)P2 in cells expressing $\operatorname{SigD} / \mathrm{SopB}$ reduced the rigidity of the plasma membrane, suggesting that local PtdIns(4,5)P2 depletion may increase susceptibility of membrane for curvature and fission (Terebiznik et al., 2002). Therefore, besides the phosphoinositide sensing PX domain, the curvature-sensing BAR domain of SNX18 is likely equally important for SNX18 recruitment to membrane invaginations and its (indirect) function in the SCV scission.

It has been reported that SopB may act on several substrates in vitro including PtdIns(3,4)P2, PtdIns(3,5)P2, PtdIns(4,5)P2, and PtdIns(3,4,5)P3 (Norris et al., 1998; Marcus et al., 2001). However, a more recent study has shown that concentration of PtdIns(3,4)P2 and PtdIns(3,4,5)P3 in $S$. Typhimurium invasion ruffles actually increases, likely by activation of class
II PI3-kinases via PtdIns(5)P as a product of SopB-mediated hydrolysis of PtdIns(4,5)P2 (Mallo et al., 2008). We propose that the transient local increase in PtdIns(3,4)P2 may be the driving cue for recruitment of SNX18, together with other phosphoinositide sensing factors like Akt. Plasma membrane recruitment of Akt is coupled with activation by phosphorylation (Steele-Mortimer et al., 2000; Marcus et al., 2001), but whether SNX18 requires activation for relocation or becomes activated upon plasma membrane recruitment still remains to be determined. A possibility remains open that to trigger SNX18 relocation the sole increase in PtdIns(3,4)P2 is not sufficient and may require an additional stimulus linked directly or indirectly to the activity of SopB.

It has been shown that upon delivery to the host cell the SopB targets SH3-containing GEF and thus can indirectly activate RhoG (Patel and Galan, 2006) that promotes Racl activation and membrane ruffling. Although we demonstrate that SopBmediated recruitment of SNX18 to the plasma membrane occurs independently or upstream of Rac1 activation (Figures S2, S3) together with recruitment of Dynamin-2 and N-WASP, it remains to be determined whether SNX18 or SopB are also involved in activation of the GTPase activity of Dynamin- 2 or in N-WASP activation of Arp2/3 machinery.

The study we present extends our understanding of $S$. Typhimurium-driven exploitation of the host molecular machinery that allows it to invade and replicate within epithelial cells. We propose that SNX18 and/or other sorting nexins are also involved in the uptake of other bacterial pathogens. It has also been reported that Enteropathogenic Escherichia coli (EPEC) secreted protein F (EspF) can directly interact with the SH3 domain of SNX9 and N-WASP to promote EPEC invasion via N-WASP/Arp2/3-mediated actin nucleation (Marches et al., 2006; Alto et al., 2007). However, EPEC induces a local increase of $\operatorname{PtdIns}(3,4,5) \mathrm{P} 3$ at the site of attachment indirectly via recruitment of phosphatidylinositol 3-kinase in a translocated intimin receptor (Tir)-dependent manner (Sason et al., 2009). Therefore, it is possible that EPEC may recruit SNX9 by an indirect mechanism similar to that of SopB-mediated recruitment of SNX18. In relation to this, the close Salmonella relative Shigella flexneri utilizes a SopB homolog (IpgD) for activation of PI3K/Akt-survival (Pendaries et al., 2006). IpgD possesses a similar activity in vitro and its intact inositol 5'-phosphatase-homology motif is essential for Akt activation (Marcus et al., 2001). During S. flexneri invasion into epithelial cells IpgD elevates the level of ruffling and macropinoscytosis in cells. However, S. flexneri does not enter cells via these induced macropinosomes but rather uses a mechanism that creates a bacterium containing vacuole $(\mathrm{BCV})$ in which the host membrane is always tightly associated with the bacteria during invasion (Weiner et al., 2016). The role of induced macropinosomes is required during the rupture of $\mathrm{BCV}$ to release $S$. flexneri into the cytoplasm (Weiner et al., 2016). Whether Shigella or other bacterial pathogens have developed a similar strategy to exploit the endocytic machinery of the host cell by recruitment of scaffolding proteins from SH3-PX-BAR sorting nexin family remains to be verified. 


\section{AUTHOR CONTRIBUTIONS}

All authors contributed to the conception, design, interpretation of the findings, drafting, and preparation of the manuscript. DL, $\mathrm{XQ}, \mathrm{YZ}$, and TB executed the experiments.

\section{FUNDING}

This work was supported by funding from the National Health and Medical Research Council (NHMRC) of Australia (606788). RT is supported by NHMRC Senior Research Fellowship (APP1041929).

\section{ACKNOWLEDGMENTS}

Fluorescent microscopy was carried out at the Australian Cancer Research Foundation (ACRF)/Institute for Molecular Bioscience (IMB) Dynamic Imaging Facility for Cancer Biology. Electron microscopy was carried out at the The Centre for Microscopy and Microanalysis (the Queensland Node of the Australian Microscopy and Microanalysis Research Facility). Services and RNAi constructs were provided by the IMB Facility for Life Science Automation (LISA). We thank to Jennifer Stow for providing Akt-PH, Rac1, and Cdc42 constructs used in this study, Brett Collins for help with design of SNX18 mutants and Seetha Karunaratne for help with constructs cloning. We also thank Sabrina Marion (Cochin Institute, University Paris Descartes) for providing FERM-EGFP construct and Jack Wang (University of Queensland) for his contribution to initiate this project.

\section{REFERENCES}

Alto, N. M., Weflen, A. W., Rardin, M. J., Yarar, D., Lazar, C. S., Tonikian, R., et al. (2007). The type III effector EspF coordinates membrane trafficking by the spatiotemporal activation of two eukaryotic signaling pathways. J. Cell Biol. 178, 1265-1278. doi: 10.1083/jcb.200705021

Aslanidis, C., and de Jong, P. J. (1990). Ligation-independent cloning of PCR products (LIC-PCR). Nucleic Acids Res. 18, 6069-6074.

Bakowski, M. A., Cirulis, J. T., Brown, N. F., Finlay, B. B., and Brumell, J. H. (2007). SopD acts cooperatively with SopB during Salmonella enterica serovar Typhimurium invasion. Cell. Microbiol. 9, 2839-2855. doi: 10.1111/j.1462-5822.2007.01000.x

Barret, C., Roy, C., Montcourrier, P., Mangeat, P., and Niggli, V. (2000). Mutagenesis of the phosphatidylinositol 4,5-bisphosphate (PIP(2)) binding site in the $\mathrm{NH}(2)$-terminal domain of ezrin correlates with its altered cellular distribution. J. Cell Biol. 151, 1067-1080. doi: 10.1083/jcb.151.5.1067

Birmingham, C. L., Smith, A. C., Bakowski, M. A., Yoshimori, T., and Brumell, J. H. (2006). Autophagy controls Salmonella infection in response to damage to the Salmonella-containing vacuole. J. Biol. Chem. 281, 11374-11383. doi: 10.1074/jbc.M509157200

Boyle, E. C., Brown, N. F., and Finlay, B. B. (2006). Salmonella enterica serovar Typhimurium effectors SopB, SopE, SopE2 and SipA disrupt tight junction structure and function. Cell. Microbiol. 8, 1946-1957. doi: 10.1111/j.1462-5822.2006.00762.x

Braun, V., Wong, A., Landekic, M., Hong, W. J., Grinstein, S., and Brumell, J. H. (2010). Sorting nexin 3 (SNX3) is a component of a tubular endosomal network induced by Salmonella and involved in maturation of the Salmonella-containing vacuole. Cell. Microbiol. 12, 1352-1367. doi: $10.1111 /$ j.1462-5822.2010.01476.x

\section{SUPPLEMENTARY MATERIAL}

The Supplementary Material for this article can be found online at: http://journal.frontiersin.org/article/10.3389/fcimb. 2017.00257/full\#supplementary-material

Movie 1 | SNX18 (EGFP) recruitment from cytosol to the plasma membrane during S. Typhimurium (mRFP) invasion. Zoom: Transient SNX18 recruitment to the nascent SCV in infected cell (1) and to the nascent macropinosomes in non-infected cell (2). HEK293 cells with stable expression of EGFP-SNX18, 1-5 min post infection. (0.1 min between frames, $7 \mathrm{fps}$ ).

Movie 2 | SNX18 (EGFP) recruits to the edge of Salmonella-induced membrane ruffles and accumulates on the nascent SCV during its scission from the plasma membrane. HEK293 cells with stable expression of EGFP-SNX18, 1-5 min post infection. (0.1 min between frames, $7 \mathrm{fps}$ ).

Movie 3 | SNX18 (EGFP) recruits to the site of S. Typhimurium (mRFP) attachment at the plasma membrane and accumulates during bacteria internalization into the nascent SCV. HEK293 cells, $16 \mathrm{~h}$ post transfection, 1-5 min post infection. ( $0.1 \mathrm{~min}$ between frames, $7 \mathrm{fps}$ ).

Movie 4 | SNX18: $\triangle \mathrm{SH} 3$ (EGFP) recruits to the site of S. Typhimurium (RFP) attachment at the plasma membrane and accumulates during bacteria internalization into the nascent SCV. HEK293 cells, $16 \mathrm{~h}$ post transfection, 1-5 min post infection. (0.3 min between frames, $7 \mathrm{fps}$ ).

Movie 5 | SNX18:R303Q (EGFP) remains cytosolic during S. Typhimurium (RFP) attachment to the plasma membrane and prolonged internalization into the nascent SCV. HEK293 cells, $16 \mathrm{~h}$ post transfection, 1-5 min post infection. (0.1 min between frames, $7 \mathrm{fps}$ ).

Movie 6 | Transient burst of SNX18 (mCherry) at the site of S. Typhimurium internalization occurs simultaneously with the Akt-PH and TAPP1-PH (EGFP), whereas no increase in Btk-PH (EGFP) signal was detected. HEK293 cells, $8 \mathrm{~h}$ post co-transfection, 1-5 min post infection. (0.2 min between frames, $7 \mathrm{fps}$ ).

Bryant, D. M., Kerr, M. C., Hammond, L. A., Joseph, S. R., Mostov, K. E., Teasdale, R. D., et al. (2007). EGF induces macropinocytosis and SNX1modulated recycling of E-cadherin. J. Cell Sci. 120(Pt 10), 1818-1828. doi: $10.1242 /$ jcs.000653

Bujny, M. V., Ewels, P. A., Humphrey, S., Attar, N., Jepson, M. A., and Cullen, P. J. (2008). Sorting nexin-1 defines an early phase of Salmonellacontaining vacuole-remodeling during Salmonella infection. J. Cell Sci. 121(Pt 12), 2027-2036. doi: 10.1242/jcs.018432

Cain, R. J., Hayward, R. D., and Koronakis, V. (2008). Deciphering interplay between Salmonella invasion effectors. PLoS Pathog. 4:e1000037. doi: 10.1371/journal.ppat.1000037.

Clark, L., Perrett, C. A., Malt, L., Harward, C., Humphrey, S., Jepson, K. A., et al. (2011). Differences in Salmonella enterica serovar Typhimurium strain invasiveness are associated with heterogeneity in SPI-1 gene expression. Microbiology 157(Pt 7), 2072-2083. doi: 10.1099/mic.0.048496-0

Dowler, S., Currie, R. A., Campbell, D. G., Deak, M., Kular, G., Downes, C. P., et al. (2000). Identification of pleckstrin-homology-domain-containing proteins with novel phosphoinositide-binding specificities. Biochem. J. 351(Pt 1), 19-31. doi: 10.1042/bj3510019

Drecktrah, D., Knodler, L. A., and Steele-Mortimer, O. (2004). Modulation and utilization of host cell phosphoinositides by Salmonella spp. Infect. Immun. 72, 4331-4335. doi: 10.1128/IAI.72.8.4331-4335.2004

Finlay, B. B., Ruschkowski, S., and Dedhar, S. (1991). Cytoskeletal rearrangements accompanying Salmonella entry into epithelial-cells. J. Cell Sci. 99, 283-296.

Forsberg, M., Blomgran, R., Lerm, M., Sarndahl, E., Sebti, S. M., Hamilton, A., et al. (2003). Differential effects of invasion by and phagocytosis of Salmonella typhimurium on apoptosis in human macrophages: potential role of Rho-GTPases and Akt. J. Leukoc. Biol. 74, 620-629. doi: 10.1189/jlb.12 02586 
Francis, C. L., Ryan, T. A., Jones, B. D., Smith, S. J., and Falkow, S. (1993). Ruffles induced by Salmonella and other stimuli direct macropinocytosis of bacteria. Nature 364, 639-642. doi: 10.1038/364639a0

Haberg, K., Lundmark, R., and Carlsson, S. R. (2008). SNX18 is an SNX9 paralog that acts as a membrane tubulator in AP-1-positive endosomal trafficking. J. Cell Sci. 121(Pt 9), 1495-1505. doi: 10.1242/jcs.028530

Haglund, C. M., and Welch, M. D. (2011). Pathogens and polymers: microbehost interactions illuminate the cytoskeleton. J. Cell Biol. 195, 7-17. doi: $10.1083 /$ jcb. 201103148

Hanisch, J., Kolm, R., Wozniczka, M., Bumann, D., Rottner, K., and Stradal, T. E. (2011). Activation of a RhoA/myosin II-dependent but Arp2/3 complexindependent pathway facilitates Salmonella invasion. Cell Host Microbe 9, 273-285. doi: 10.1016/j.chom.2011.03.009

Haraga, A., Ohlson, M. B., and Miller, S. I. (2008). Salmonellae interplay with host cells. Nat. Rev. Microbiol. 6, 53-66. doi: 10.1038/nrmicro1788

Hernandez, L. D., Hueffer, K., Wenk, M. R., and Galan, J. E. (2004). Salmonella modulates vesicular traffic by altering phosphoinositide metabolism. Science 304, 1805-1807. doi: 10.1126/science.1098188304/5678/1805

James, S. R., Downes, C. P., Gigg, R., Grove, S. J., Holmes, A. B., and Alessi, D. R. (1996). Specific binding of the Akt-1 protein kinase to phosphatidylinositol 3,4,5-trisphosphate without subsequent activation. Biochem. J. 315 (Pt 3), $709-713$

Kerr, M. C., C. N., Karunaratne, S., and Teasdale, R. D. (2012). "The phosphoinositides: key regulators of Salmonella Containing Vacuole (SCV) trafficking and identity," in Salmonella-Distribution, Adaptation, Control Measures and Molecular Technologies, ed G. J. Annous (Rijeka: BA. InTech), 251-264.

Kerr, M. C., Wang, J. T., Castro, N. A., Hamilton, N. A., Town, L., Brown, D. L., et al. (2010). Inhibition of the PtdIns(5) kinase PIKfyve disrupts intracellular replication of Salmonella. EMBO J. 29, 1331-1347. doi: 10.1038/emboj.2010.28

Knodler, L. A., Bestor, A., Ma, C., Hansen-Wester, I., Hensel, M., Vallance, B. A., et al. (2005). Cloning vectors and fluorescent proteins can significantly inhibit Salmonella enterica virulence in both epithelial cells and macrophages: implications for bacterial pathogenesis studies. Infect. Immun. 73, 7027-7031. doi: 10.1128/IAI.73.10.7027-7031.2005

Kupz, A., Guarda, G., Gebhardt, T., Sander, L. E., Short, K. R., Diavatopoulos, D. A., et al. (2012). NLRC4 inflammasomes in dendritic cells regulate noncognate effector function by memory $\mathrm{CD}^{+} \mathrm{T}$ cells. Nat. Immunol. 13, 162-169. doi: 10.1038/ni.2195

Lim, J. P., Wang, J. T., Kerr, M. C., Teasdale, R. D., and Gleeson, P. A. (2008). A role for SNX5 in the regulation of macropinocytosis. BMC Cell Biol. 9:58. doi: 10.1186/1471-2121-9-58

Lommel, S., Benesch, S., Rottner, K., Franz, T., Wehland, J., and Kuhn, R. (2001). Actin pedestal formation by enteropathogenic Escherichia coli and intracellular motility of Shigella flexneri are abolished in N-WASP-defective cells. EMBO Rep. 2, 850-857. doi: 10.1093/embo-reports/kve1972/9/850

Mallo, G. V., Espina, M., Smith, A. C., Terebiznik, M. R., Aleman, A., Finlay, B. B., et al. (2008). SopB promotes phosphatidylinositol 3-phosphate formation on Salmonella vacuoles by recruiting Rab5 and Vps34. J. Cell Biol. 182, 741-752. doi: $10.1083 /$ jcb.200804131

Marches, O., Batchelor, M., Shaw, R. K., Patel, A., Cummings, N., Nagai, T., et al. (2006). EspF of enteropathogenic Escherichia coli binds sorting nexin 9. J. Bacteriol. 188, 3110-3115. doi: 10.1128/JB.188.8.3110-3115.2006

Marcus, S. L., Wenk, M. R., Steele-Mortimer, O., and Finlay, B. B. (2001). A synaptojanin-homologous region of Salmonella typhimurium SigD is essential for inositol phosphatase activity and Akt activation. FEBS Lett. 494, 201-207. doi: S0014-5793(01)02356-0

Marion, S., Hoffmann, E., Holzer, D., Le Clainche, C., Martin, M., Sachse, M., et al. (2011). Ezrin promotes actin assembly at the phagosome membrane and regulates phago-lysosomal fusion. Traffic 12, 421-437. doi: 10.1111/j.1600-0854.2011.01158.x

Mason, D., Mallo, G. V., Terebiznik, M. R., Payrastre, B., Finlay, B. B., Brumell, J. H., et al. (2007). Alteration of epithelial structure and function associated with PtdIns(4,5)P2 degradation by a bacterial phosphatase. J. Gen. Physiol. 129, 267-283. doi: 10.1085/jgp.200609656

Nakazawa, S., Gotoh, N., Matsumoto, H., Murayama, C., Suzuki, T., and Yamamoto, T. (2011). Expression of sorting nexin 18 (SNX18) is dynamically regulated in developing spinal motor neurons. J. Histochem. Cytochem. 59, 202-213. doi: 10.1369/0022155410392231

Norris, F. A., Wilson, M. P., Wallis, T. S., Galyov, E. E., and Majerus, P. W. (1998) SopB, a protein required for virulence of Salmonella dublin, is an inositol phosphate phosphatase. Proc. Natl. Acad. Sci. U.S.A. 95, 14057-14059.

Park, J., Kim, Y., Lee, S., Park, J. J., Park, Z. Y., Sun, W., et al. (2010). SNX18 shares a redundant role with SNX9 and modulates endocytic trafficking at the plasma membrane. J. Cell Sci. 123(Pt 10), 1742-1750. doi: 10.1242/jcs.064170

Patel, J. C., and Galan, J. E. (2006). Differential activation and function of Rho GTPases during Salmonella-host cell interactions. J. Cell Biol. 175, 453-463. doi: $10.1083 /$ jcb.200605144

Pattni, K., Jepson, M., Stenmark, H., and Banting, G. (2001). A PtdIns(3)P-specific probe cycles on and off host cell membranes during Salmonella invasion of mammalian cells. Curr. Biol. 11, 1636-1642. doi: 10.1016/S0960-9822(01)00486-9

Pendaries, C., Tronchere, H., Arbibe, L., Mounier, J., Gozani, O., Cantley, L., et al. (2006). PtdIns5P activates the host cell PI3-kinase/Akt pathway during Shigella flexneri infection. EMBO J. 25, 1024-1034. doi: 10.1038/sj.emboj.7601001

Piper, R. C., Hess, L. J., and James, D. E. (1991). Differential sorting of two glucose transporters expressed in insulin-sensitive cells. Am. J. Physiol. 260(3 Pt 1), C570-C580.

Piscatelli, H. L., Li, M. H., and Zhou, D. G. (2016). Dual 4-and 5-phosphatase activities regulate SopB-dependent phosphoinositide dynamics to promote bacterial entry. Cell. Microbiol. 18, 705-719. doi: 10.1111/cmi.12542

Pizarro-Cerda, J., and Cossart, P. (2004). Subversion of phosphoinositide metabolism by intracellular bacterial pathogens. Nat. Cell Biol. 6, 1026-1033. doi: $10.1038 /$ ncb1104-1026

Pylypenko, O., Lundmark, R., Rasmuson, E., Carlsson, S. R., and Rak, A. (2007). The PX-BAR membrane-remodeling unit of sorting nexin 9. EMBO J. 26, 4788-4800. doi: 10.1038/sj.emboj.7601889

Riedl, J., Crevenna, A. H., Kessenbrock, K., Yu, J. H., Neukirchen, D., Bista, M. et al. (2008). Lifeact: a versatile marker to visualize F-actin. Nat. Methods 5 605-607. doi: 10.1038/nmeth.1220

Salim, K., Bottomley, M. J., Querfurth, E., Zvelebil, M. J., Gout, I., Scaife, R., et al. (1996). Distinct specificity in the recognition of phosphoinositides by the pleckstrin homology domains of dynamin and Bruton's tyrosine kinase. $E M B O$ J. 15, 6241-6250.

Sason, H., Milgrom, M., Weiss, A. M., Melamed-Book, N., Balla, T., Grinstein, S., et al. (2009). Enteropathogenic Escherichia coli subverts phosphatidylinositol 4,5-bisphosphate and phosphatidylinositol 3,4,5-trisphosphate upon epithelial cell infection. Mol. Biol. Cell 20, 544-555. doi: 10.1091/mbc.E08-05-0516

Shin, N., Ahn, N., Chang-Ileto, B., Park, J., Takei, K., Ahn, S. G., et al. (2008). SNX9 regulates tubular invagination of the plasma membrane through interaction with actin cytoskeleton and dynamin 2. J. Cell Sci. 121(Pt 8), 1252-1263. doi: $10.1242 /$ jcs.016709

Soulet, F., Yarar, D., Leonard, M., and Schmid, S. L. (2005). SNX9 regulates dynamin assembly and is required for efficient clathrin-mediated endocytosis. Mol. Biol. Cell 16, 2058-2067. doi: 10.1091/mbc.E04-11-1016

Steele-Mortimer, O., Knodler, L. A., Marcus, S. L., Scheid, M. P., Goh, B., Pfeifer, C. G., et al. (2000). Activation of Akt/protein kinase B in epithelial cells by the Salmonella typhimurium effector sigD. J. Biol. Chem. 275, 37718-37724. doi: 10.1074/jbc.M008187200

Stender, S., Friebel, A., Linder, S., Rohde, M., Mirold, S., and Hardt, W. D. (2000). Identification of SopE2 from Salmonella typhimurium, a conserved guanine nucleotide exchange factor for Cdc42 of the host cell. Mol. Microbiol. 36, 1206-1221. doi: 10.1046/j.1365-2958.2000.01933.x

Teasdale, R. D., and Collins, B. M. (2012). Insights into the PX (phox-homology) domain and SNX (sorting nexin) protein families: structures, functions and roles in disease. Biochem. J. 441, 39-59. doi: 10.1042/BJ20111226

Terebiznik, M. R., Vieira, O. V., Marcus, S. L., Slade, A., Yip, C. M., Trimble, W. S., et al. (2002). Elimination of host cell PtdIns(4,5)P(2) by bacterial SigD promotes membrane fission during invasion by Salmonella. Nat. Cell Biol. 4, 766-773. doi: $10.1038 / \mathrm{ncb} 854$

Unsworth, K. E., Way, M., McNiven, M., Machesky, L., and Holden, D. W. (2004). Analysis of the mechanisms of Salmonella-induced actin assembly during invasion of host cells and intracellular replication. Cell. Microbiol. 6, 1041-1055. doi: 10.1111/j.1462-5822.2004.00417.x 
van Weering, J. R., Sessions, R. B., Traer, C. J., Kloer, D. P., Bhatia, V. K., Stamou, D., et al. (2012). Molecular basis for SNX-BAR-mediated assembly of distinct endosomal sorting tubules. EMBO J. 31, 4466-4480. doi: 10.1038/emboj.2012.283

Varnai, P., Rother, K. I., and Balla, T. (1999). Phosphatidylinositol 3-kinasedependent membrane association of the Bruton's tyrosine kinase pleckstrin homology domain visualized in single living cells. J. Biol. Chem. 274, 10983-10989.

Veiga, E., Guttman, J. A., Bonazzi, M., Boucrot, E., Toledo-Arana, A., Lin, A. E., et al. (2007). Invasive and adherent bacterial pathogens co-Opt host clathrin for infection. Cell Host Microbe 2, 340-351. doi: 10.1016/j.chom.2007.10.001

Wang, B., Wylie, F. G., Teasdale, R. D., and Stow, J. L. (2005). Polarized trafficking of E-cadherin is regulated by Racl and Cdc42 in MadinDarby canine kidney cells. Am. J. Physiol. Cell Physiol. 288, C1411-C1419. doi: 10.1152/ajpcell.00533.2004

Wang, J. T., Kerr, M. C., Karunaratne, S., Jeanes, A., Yap, A. S., and Teasdale, R. D. (2010). The SNX-PX-BAR family in macropinocytosis: the regulation of macropinosome formation by SNX-PX-BAR proteins. PLOS ONE 5:e13763. doi: 10.1371/journal.pone.0013763

Weiner, A., Mellouk, N., Lopez-Montero, N., Chang, Y. Y., Souque, C., Schmitt, C., et al. (2016). Macropinosomes are key players in early shigella invasion and vacuolar escape in epithelial cells. PLoS Pathog. 12:e1005602. doi: 10.1371/journal.ppat.1005602

Yarar, D., Surka, M. C., Leonard, M. C., and Schmid, S. L. (2008). SNX9 activities are regulated by multiple phosphoinositides through both PX and BAR domains. Traffic 9, 133-146. doi: 10.1111/j.1600-0854.2007.00 675.x

Zhang, J., Zhang, X., Guo, Y., Xu, L., and Pei, D. (2009). Sorting nexin 33 induces mammalian cell micronucleated phenotype and actin polymerization by interacting with Wiskott-Aldrich syndrome protein. J. Biol. Chem. 284, 21659-21669. doi: 10.1074/jbc.M109.007278

Conflict of Interest Statement: The authors declare that the research was conducted in the absence of any commercial or financial relationships that could be construed as a potential conflict of interest.

Copyright (C) 2017 Liebl, Qi, Zhe, Barnett and Teasdale. This is an open-access article distributed under the terms of the Creative Commons Attribution License (CC BY). The use, distribution or reproduction in other forums is permitted, provided the original author(s) or licensor are credited and that the original publication in this journal is cited, in accordance with accepted academic practice. No use, distribution or reproduction is permitted which does not comply with these terms. 